\title{
COSMIC DEFECTS AND COSMOLOGY
}

\author{
JOÃO MAGUEIJO \\ Theoretical Physics, The Blackett Laboratory, Imperial College \\ Prince Consort Road, London SW7 2BZ, UK \\ AND \\ ROBERT H. BRANDENBERGER \\ Physics Department, Brown University \\ Providence, RI, 02912, USA
}

\begin{abstract}
We provide a pedagogical overview of defect models of structure formation. We first introduce the concept of topological defect, and describe how to classify them. We then show how defects might be produced in phase transitions in the Early Universe and approach non-pathological scaling solutions. A very heuristic account of structure formation with defects is then provided, following which we introduce the tool box required for high precision calculations of CMB and LSS power spectra in these theories. The decomposition into scalar vector and tensor modes is reviewed, and then we introduce the concept of unequal-time correlator. We use isotropy and causality to constrain the form of these correlators. We finally show how these correlators may be decomposed into eigenmodes, thereby reducing a defect problem to a series of "inflation" problems. We conclude with a short description of results in these theories and how they fare against observations. We finally describe yet another application of topological defects in cosmology: baryogenesis.
\end{abstract}

\section{Introduction}

Phase transitions are ubiquitous in nature. Typically, as the temperature of a system drops below the critical temperature, the system makes a transition from a state with greater symmetry to one with less symmetry. In general, the state with less symmetry is not unique, but can lie anywhere in a so-called vacuum manifold. Depending on the topology of this vacuum 
manifold, defects will form during the phase transition. If the topology of the vacuum manifold admits defects, then these defects will inevitably arise during the phase transition unless the dynamics is completely adiabatic.

In the early Universe the temperature was decreasing very rapidly. On length scales larger than the Hubble radius, causality prevents the system from maintaining adiabaticity through interactions, and therefore on these scales defects will arise in any cosmological phase transition in which they are topologically allowed. The Universe has undergone several phase transitions. We are quite confident about those which occurred at lower temperatures: the confinement transition at a temperature $T \sim 10^{2} \mathrm{GeV}$ and the electroweak symmetry breaking transition at $T \sim 10^{3} \mathrm{GeV}$. Unified field theories of fundamental interactions predict the existence of other transitions at higher temperatures, e.g. a phase transition at $T \sim 10^{16} \mathrm{GeV}$ in Grand Unified Models, the supersymmetry breaking phase transition in supersymmetric models, and various compactification transitions in string (and M-) theory.

Since topological defects carry energy density, they will curve spacetime and can thus act as the seeds for gravitational accretion (see e.g. Refs. [1, 2, 3, 4, 5] for comprehensive reviews). Since inside of topological defects the symmetry characteristic of the high temperature phase is unbroken, topological defects can interact in various interesting ways with the surrounding matter and can have an effect on cosmological issues such as baryogenesis (see e.g. $[6,7,8]$ ), magnetic field generation (see e.g. $[9,10]$ ), and ultra-high-energy cosmic ray production $[11,12,13,14]$.

In these lectures, we first review the classification of topological defects and explain why in models with the appropriate topology, defects will inevitably form during the symmetry breaking phase transition. In Section 3 we discuss some initial applications of topological defects to cosmology. We review the domain wall and monopole problems and explain why the cosmic string model yields a promising mechanism for structure formation.

In the following sections we provide a more technical description of how high accuracy calculations of structure formation in defect theories are performed. We first describe the details of the scalar, vector and tensor decomposition (Section 6). This is an invaluable tool in linear perturbation theory. Then in Section 7 we introduce the concept of UETC and show their general form, assuming isotropy and scaling, but not energy conservation. In Section 8 we show how causality limits further the form of the correlators, in the large wavelength limit. These results will be important when checking upon the numerics. Then in Section 11 we present the UETCs measured for cosmic strings, and highlight some of their features. Their remarkable novelty is the dominance of the energy density over any other components of the stress energy tensor. This property sets strings apart, resulting in 
a dominance of scalar modes over vector and tensor modes. We present some conclusions on string scenarios of structure formation, and also hybrid scenarios combining strings and inflation.

In the final section we illustrate one application of topological defects to cosmology which involves microscopic physics rather than gravitational accretion: we discuss the basics of defect-mediated baryogenesis.

\section{Defect Classification and Formation}

According to our current particle physics theories, matter at high energies and temperatures must be described in terms of fields. Gauge symmetries have proved to be extremely useful in describing the standard model of particle physics, according to which at high energies the laws of nature are invariant under some non-abelian group of internal symmetry transformations $G=\mathrm{SU}(3)_{c} \times \mathrm{SU}(2)_{L} \times U(1)_{Y}$, where the first factor is the symmetry group of the strong interactions, and the second and third factors form the gauge group of the Glashow-Weinberg-Salam theory of electroweak interactions which is spontaneously broken to the $U(1)$ of electromagnetism at a scale of $T \sim 10^{3} \mathrm{GeV}$.

Spontaneous symmetry breaking is induced by an order parameter $\varphi$ taking on a nontrivial expectation value $\langle\varphi\rangle$ below a certain temperature $T_{c}$. In some particle physics models, $\varphi$ is a fundamental scalar field in a nontrivial representation of the gauge group $G$. However, $\varphi$ could also be a fermion condensate, as in the BCS theory of superconductivity.

The transition taking place at $T=T_{c}$ is a phase transition and $T_{c}$ is called the critical temperature. From condensed matter physics it is well known that in many cases topological defects form during phase transitions, particularly if the transition rate is fast on a scale compared to the system size. When cooling a metal, defects in the crystal configuration will be frozen in; during a temperature quench of ${ }^{4} \mathrm{He}$, thin vortex tubes of the normal phase are trapped in the superfluid; and analogously in a temperature quench of a superconductor, flux lines are trapped in a surrounding sea of the superconducting Meissner phase.

In cosmology, the rate at which the phase transition proceeds is given by the expansion rate of the Universe. Hence, topological defects will inevitably be produced in a cosmological phase transition [15], provided the underlying particle physics model allows such defects.

Topological defects can be point-like (monopoles), string-like (cosmic strings) [16] or planar (domain walls), depending on the particle physics model. Also of importance are textures [17, 18], point defects in space-time. Topological defects represent regions in space with trapped energy density. These regions of surplus energy can act as seeds for structure formation. 
Consider a single component real scalar field with a typical symmetry breaking potential

$$
V(\varphi)=\frac{1}{4} \lambda\left(\varphi^{2}-\eta^{2}\right)^{2}
$$

Unless $\lambda \ll 1$ there will be no inflation. The phase transition will take place on a short time scale $\tau<H^{-1}$, and will lead to correlation regions of radius $\xi<t$ inside of which $\varphi$ is approximately constant, but outside of which $\varphi$ ranges randomly over the vacuum manifold $\mathcal{M}$, the set of values of $\varphi$ which minimizes $V(\varphi)$ - in our example $\varphi= \pm \eta$. The correlation regions are separated by domain walls, regions in space where $\varphi$ leaves the vacuum manifold $\mathcal{M}$ and where, therefore, potential energy is localized. Via the usual gravitational force, this energy density can act as a seed for structure.

As mentioned above, topological defects are familiar from solid state and condensed matter systems. The analogies between defects in particle physics and condensed matter physics are quite deep. Defects form for the same reason: the vacuum manifold is topologically nontrivial. The arguments which say that in a theory which admits defects, such defects will inevitably form, are applicable both in cosmology and in condensed matter physics. Different, however, is the defect dynamics. The motion of defects in condensed matter systems is friction-dominated, whereas the defects in cosmology obey relativistic equations, second order in time derivatives, since they come from a relativistic field theory.

Turning to a classification of topological defects, we consider theories with an $n$-component order parameter $\varphi$ and with a potential energy function (free energy density) of the form (1) with

$$
\varphi^{2}=\sum_{i=1}^{n} \varphi_{i}^{2} .
$$

There are various types of local and global topological defects (regions of trapped energy density) depending on the number $n$ of components of $\varphi$. The more rigorous mathematical definition refers to the homotopy of $\mathcal{M}$. The words "local" and "global" refer to whether the symmetry which is broken is a gauge or global symmetry. In the case of local symmetries, the topological defects have a well defined core outside of which $\varphi$ contains no energy density in spite of nonvanishing gradients $\nabla \varphi$ : the gauge fields $A_{\mu}$ can absorb the gradients, i.e., $D_{\mu} \varphi=0$ when $\partial_{\mu} \varphi \neq 0$, where the covariant derivative $D_{\mu}$ is defined by

$$
D_{\mu}=\partial_{\mu}+i e A_{\mu},
$$

$e$ being the gauge coupling constant. Global topological defects, however, have long range density fields and forces. 
Table 1 contains a list of topological defects with their topological characteristic. A "v" marks acceptable theories, a " $\mathrm{x}$ " theories which are in conflict with observations (for $\eta \sim 10^{16} \mathrm{GeV}$ ).

TABLE 1. Classification of cosmologically allowed (v) and forbidden (x) defects.

\begin{tabular}{llll}
\hline Defect name & $\mathrm{n}$ & Local defect & Global defect \\
\hline Domain wall & 1 & $\mathrm{x}$ & $\mathrm{x}$ \\
Cosmic string & 2 & $\mathrm{v}$ & $\mathrm{v}$ \\
Monopole & 3 & $\mathrm{x}$ & $\mathrm{v}$ \\
Texture & 4 & - & $\mathrm{v}$ \\
\hline
\end{tabular}

We now describe examples of domain walls, cosmic strings, monopoles and textures, focussing on configurations with maximal symmetry.

Domain walls arise in theories with a single real order parameter and free energy density given by (1). The vacuum manifold of this model consists of two points

$$
\mathcal{M}=\{ \pm \eta\}
$$

and hence has nontrivial zeroth homotopy group:

$$
\Pi_{0}(\mathcal{M}) \neq 1
$$

To construct a domain wall configuration with planar symmetry (without loss of generality the $y-z$ plane can be taken to be the plane of symmetry), assume that

$$
\begin{gathered}
\varphi(x) \simeq \eta \quad x \gg \eta^{-1} \\
\varphi(x) \simeq-\eta \quad x \ll-\eta^{-1}
\end{gathered}
$$

By continuity of $\varphi$, there must be an intermediate value of $x$ with $\varphi(x)=0$. The set of points with $\varphi=0$ constitute the center of the domain wall. Physically, the wall is a thin sheet of trapped energy density. The width $w$ of the sheet is given by the balance of potential energy and tension energy. Assuming that the spatial gradients are spread out over the thickness $w$ we obtain

$$
w V(0)=w \lambda \eta^{4} \sim \frac{1}{w} \eta^{2}
$$

and thus

$$
w \sim \lambda^{-1 / 2} \eta^{-1}
$$


A theory with a complex order parameter $(n=2)$ admits cosmic strings. In this case the vacuum manifold of the model is

$$
\mathcal{M}=S^{1},
$$

which has nonvanishing first homotopy group:

$$
\Pi_{1}(\mathcal{M})=Z \neq 1 \text {. }
$$

A cosmic string is a line of trapped energy density which arises whenever the field $\varphi(x)$ circles $\mathcal{M}$ along a closed path in space (e.g., along a circle). In this case, continuity of $\varphi$ implies that there must be a point with $\varphi=0$ on any disk whose boundary is the closed path. The points on different sheets connect up to form a line over-density of field energy, a cosmic string.

To construct a field configuration with a string along the $z$ axis [19], take $\varphi(x)$ to cover $\mathcal{M}$ along a circle with radius $r$ about the point $(x, y)=(0,0)$ :

$$
\varphi(r, \vartheta) \simeq \eta e^{i \vartheta}, r \gg \eta^{-1} .
$$

This configuration has winding number 1 , i.e., it covers $\mathcal{M}$ exactly once. Maintaining cylindrical symmetry, we can extend (12) to arbitrary $r$

$$
\varphi(r, \vartheta)=f(r) e^{i \vartheta},
$$

where $f(0)=0$ and $f(r)$ tends to $\eta$ for large $r$. The width $w$ can again be found by balancing potential and tension energy. The result is identical to the result (9) for domain walls.

For local cosmic strings, i.e., strings arising due to the spontaneous breaking of a gauge symmetry, the energy density decays exponentially for $r \gg \eta^{-1}$. In this case, the energy $\mu$ per unit length of a string is finite and depends only on the symmetry breaking scale $\eta$

$$
\mu \sim \eta^{2}
$$

(independent of the coupling $\lambda$ ). The value of $\mu$ is the only free parameter in a cosmic string model.

If the order parameter of the model has three components $(n=3)$, then monopoles result as topological defects. The vacuum manifold is

$$
\mathcal{M}=S^{2}
$$

and has topology given by

$$
\Pi_{2}(\mathcal{M}) \neq 1 .
$$

Given a sphere $S$ in space, it is possible that $\varphi$ takes on values in $\mathcal{M}$ everywhere on $S$, and that it covers $\mathcal{M}$ once. By continuity, there must be 
a point in space in the interior of $S$ with $\varphi=0$. This is the center of a point-like defect, the monopole.

To construct a spherically symmetric monopole with the origin as its center, consider a field configuration $\varphi$ which defines a map from physical space to field space such that all spheres $S_{r}$ in space of radius $r \gg \eta^{-1}$ about the origin are mapped onto $\mathcal{M}$ :

$$
\begin{aligned}
\varphi: \quad S_{r} & \longrightarrow \mathcal{M} \\
(r, \vartheta, \varphi) & \longrightarrow(\vartheta, \varphi) .
\end{aligned}
$$

This configuration defines a winding number one magnetic monopole.

Domain walls, cosmic strings and monopoles are examples of topological defects. A topological defect has a well-defined core, a region in space with $\varphi \notin \mathcal{M}$ and hence $V(\varphi)>0$. There is an associated winding number which is quantized, i.e., it can take on only integer values. Since the winding number can only change continuously, it must be conserved, and hence topological defects are stable. Furthermore, topological defects exist for theories with global and local symmetries.

Now, let us consider a theory with a four-component order parameter (i.e., $n=4$ ), and a potential given by (1). In this case, the vacuum manifold is

$$
\mathcal{M}=S^{3}
$$

and the associated topology is given by

$$
\Pi_{3}(\mathcal{M}) \neq 1
$$

The corresponding defects are called textures [17, 18].

Textures, however, are quite different than the previously discussed topological defects. The texture construction will render this manifest. To construct a radially symmetric texture, we give a field configuration $\varphi(x)$ which maps physical space onto $\mathcal{M}$. The origin 0 in space (an arbitrary point which will be the center of the texture) is mapped onto the north pole $N$ of $\mathcal{M}$. Spheres in space of radius $r$ surrounding 0 are mapped onto spheres in $\mathcal{M}$ surrounding $N$, with distance from $N$ increasing as $r$ increases. In particular, some sphere with radius $r_{c}(t)$ is mapped onto the equator sphere of $\mathcal{M}$. The distance $r_{c}(t)$ can be defined as the radius of the texture. Inside this sphere, $\varphi(x)$ covers half the vacuum manifold. Finally, the sphere at infinity is mapped onto the south pole of $\mathcal{M}$. The configuration $\varphi(x)$ can be parameterized by [18]

$$
\varphi(x, y, z)=\left(\cos \chi(r), \sin \chi(r) \frac{x}{r}, \sin \chi(r) \frac{y}{r}, \sin \chi(r) \frac{z}{r}\right)
$$


in terms of a function $\chi(r)$ with $\chi(0)=0$ and $\chi(\infty)=\pi$. Note that at all points in space, $\varphi(x)$ lies in $\mathcal{M}$. There is no defect core. All the energy is in spatial gradient (and possibly kinetic) terms.

In a cosmological context, there is infinite energy available in an infinite space. Hence, it is not necessary that $\chi(r) \rightarrow \pi$ as $r \rightarrow \infty$. We can have

$$
\chi(r) \rightarrow \chi_{\max }<\pi \text { as } r \rightarrow \infty .
$$

In this case, only a fraction

$$
n_{W}=\frac{\chi_{\max }}{\pi}-\frac{\sin \left(2 \chi_{\max }\right)}{2 \pi}
$$

of the vacuum manifold is covered: the winding number $n_{W}$ is not quantized. This is a reflection of the fact that whereas topologically nontrivial maps from $S^{3}$ to $S^{3}$ exist, all maps from $R^{3}$ to $S^{3}$ can be deformed to the trivial map.

Textures in $R^{3}$ are unstable. For the configuration described above, the instability means that $r_{c}(t) \rightarrow 0$ as $t$ increases: the texture collapses. When $r_{c}(t)$ is microscopical, there will be sufficient energy inside the core to cause $\varphi(0)$ to leave $\mathcal{M}$, pass through 0 and equilibrate at $\chi(0)=\pi$ : the texture unwinds.

A further difference compared to topological defects: textures are relevant only for theories with global symmetry. Since all the energy is in spatial gradients, for a local theory the gauge fields can re-orient themselves such as to cancel the energy:

$$
D_{\mu} \varphi=0 .
$$

Therefore, it is reasonable to regard textures as an example of a new class of defects, semi-topological defects. In contrast to topological defects, there is no core, and $\varphi(x) \in \mathcal{M}$ for all $x$. In particular, there is no potential energy. In addition, the winding number is not quantized, and hence the defects are unstable. Finally, they exist as long-lived coherent configurations only in theories with a global internal symmetry.

The Kibble mechanism [15] ensures that in theories which admit topological or semi-topological defects, such defects will be produced during a phase transition in the very early Universe. At high temperatures $T \gg T_{c}$, the symmetry is unbroken. The ground state of the finite temperature effective potential $[20,21]$ (see e.g. [22] for an introductory review) is the symmetric configuration $\varphi=0$. Once the Universe cools below the temperature $T_{c}$, the symmetry is broken, and $\varphi(x)$ rolls into the zero temperature vacuum manifold $\mathcal{M}$. However, by causality there can be no correlation between the specific points in $\mathcal{M}$ which are taken on on scales larger than 
the correlation length $\xi(t)$. In a relativistic theory the causality bound on $\xi$ is

$$
\xi(t)<t,
$$

where $t$ is the causal horizon. This leads to the formation of defects with a mean separation of $\xi(t)$.

The correlation length $\xi(t)$ can be determined by equating the free energy gained by symmetry breaking (a volume effect) with the gradient energy lost (a surface effect). As expected, $\xi(T)$ diverges at $T_{c}$. Very close to $T_{c}$, the thermal energy $T$ is larger than the volume energy gain $E_{c o r r}$ in a correlation volume. Hence, these domains are unstable to thermal fluctuations. As $T$ decreases, the thermal energy decreases more rapidly than $E_{\text {corr }}$. Below the Ginsburg temperature $T_{G}$, there is insufficient thermal energy to excite a correlation volume into the state $\varphi=0$. Domains of size

$$
\xi\left(t_{G}\right) \sim \lambda^{-1} \eta^{-1}
$$

freeze out $[15,23]$. The boundaries between these domains become topological defects. An improved version of this argument has been given by Zurek [24].

We conclude that in a theory in which a symmetry breaking phase transition satisfies the topological criteria for the existence of a given type of defect, a network of such defects will form during the phase transition and will freeze out at the Ginsburg temperature. The correlation length is initially given by (26), if the field $\varphi$ is in thermal equilibrium before the transition. Independent of this last assumption, the causality bound implies that $\xi\left(t_{G}\right)<t_{G}$.

For times $t>t_{G}$ the evolution of the network of defects may be complicated (as for cosmic strings) or trivial (as for textures). In any case (see the caveats of Refs. [25, 26]), the causality bound persists at late times and states that even at late times, the mean separation and length scale of defects is bounded by $\xi(t) \leq t$.

Applied to cosmic strings, the Kibble mechanism implies that at the time of the phase transition, a network of cosmic strings with typical step length $\xi\left(t_{G}\right)$ will form. According to numerical simulations [27], about $80 \%$ of the initial energy is in infinite strings (strings with curvature radius larger than the Hubble radius) and $20 \%$ in closed loops.

The evolution of the cosmic string network for $t>t_{G}$ is complicated. The key processes are loop production by intersections of infinite strings and loop shrinking by gravitational radiation. These two processes combine to create a mechanism by which the infinite string network loses energy (and length as measured in comoving coordinates). The dynamics of a string in an expanding Universe changes as the curvature radius increases. If the 
curvature radius is smaller than the Hubble radius $H^{-1}(t)$, the dynamics is dominated by the acceleration term and the string will oscillate at relativistic speeds. However, if the curvature radius exceeds the Hubble radius, the strings are frozen in by the Hubble damping term in the string equation of motion. Therefore, if the string correlation length is smaller than the Hubble radius, string intersections will be frequent, the network will loose a lot of energy to loops and $\xi(t)$ will increase faster than $t$. However, if at any time $\xi(t)$ exceeds the Hubble radius, the strings will be effectively frozen in comoving coordinates and hence in a standard radiation or matter dominated cosmology the Hubble radius will catch up to $\xi(t)$. According to this argument [2], at sufficiently late times the correlation leng! th of the string network will always be proportional to its causality limit

$$
\xi(t) \sim t
$$

Hence, the energy density $\rho_{\infty}(t)$ in long strings is a fixed fraction of the background energy density $\rho_{c}(t)$

$$
\rho_{\infty}(t) \sim \mu \xi(t)^{-2} \sim \mu t^{-2}
$$

or

$$
\frac{\rho_{\infty}(t)}{\rho_{c}(t)} \sim G \mu
$$

We conclude that the cosmic string network approaches a "scaling solution" in which the statistical properties of the network are time independent if all distances are scaled to the Hubble radius.

Applied to textures (see e.g. [28] for a review), the Kibble mechanism implies that on all scales $r \geq t_{G}$, field configurations with winding number $n_{W} \geq n_{c r}$ are frozen in with a probability $p\left(n_{c r}\right)$ per volume $r^{3}$. The critical winding number $n_{c r}$ is defined as the winding number above which field configurations collapse and below which they expand. Only collapsing configurations form clumps of energy which can accrete matter. For spherically symmetric textures, the critical winding $n_{c r}$ is slightly larger than 0.5 [29].

For $t>t_{G}$, any configuration on scale $\sim t$ with winding number $n_{W} \geq$ $n_{c r}$ begins to collapse (if the scale of the texture is larger than $t$, the Hubble damping term dominates over the spatial gradient forces, and the field configuration is frozen in comoving coordinates). After unwinding, $\varphi(x)$ is homogeneous inside the horizon.

The texture model thus also leads to a scaling solution: at all times $t>t_{G}$ there is the same probability that a texture configuration of scale $t$ will enter the horizon, become dynamical and collapse on a typical time scale $t$. 


\section{Defects and Structure Formation: An Overview}

Topological defects are regions in space with trapped energy density. By Newtonian gravity, these defects can act as seeds about which the matter in the Universe clusters, and hence they play a very important role in cosmology.

As indicated in Table 1, theories with domain walls or with local monopoles are ruled out, and those with only local textures do not give rise to a structure formation model. As mentioned earlier, theories with domain walls are ruled out since a single wall stretching across the present Universe would overclose it. Local monopoles are also problematic since they do not interact and come to dominate the energy density of the Universe. Local textures do not exist as coherent structures with nonvanishing gradient energy since the gauge fields can always compensate scalar field gradients.

Let us demonstrate explicitly why stable domain walls are a cosmological disaster [30]. If domain walls form during a phase transition in the early Universe, it follows by causality (see however the caveats of Refs. [25, 26]) that even today there will be at least one wall per Hubble volume. Assuming one wall per Hubble volume, the energy density $\rho_{D W}$ of matter in domain walls is

$$
\rho_{D W}(t) \sim \eta^{3} t^{-1}
$$

whereas the critical density $\rho_{c}$ is

$$
\rho_{c}=H^{2} \frac{3}{8 \pi G} \sim m_{p \ell}^{2} t^{-2} .
$$

Hence, for $\eta \sim 10^{16} \mathrm{GeV}$ the ratio evaluated at the present time $t_{0}$ is

$$
\frac{\rho_{D W}}{\rho_{c}}\left(t_{0}\right) \sim\left(\frac{\eta}{m_{p \ell}}\right)^{2}\left(\eta t_{0}\right) \sim 10^{52} .
$$

The above argument depends in an essential way on the dimension of the defect. One cosmic string per Hubble volume leads to an energy density $\rho_{c s}$ in string

$$
\rho_{c s} \sim \eta^{2} t^{-2} .
$$

As we have seen above, the cosmic string network approaches a scaling distribution (27). Hence, cosmic strings do not lead to cosmological problems. On the contrary, since for GUT models with $\eta \sim 10^{16} \mathrm{GeV}$

$$
\frac{\rho_{c s}}{\rho_{c}} \sim\left(\frac{\eta}{m_{p \ell}}\right)^{2} \sim 10^{-6},
$$

cosmic strings in such theories could provide the seed perturbations responsible for structure formation. 
Theories with local monopoles are ruled out on cosmological grounds [31] (see again the caveats of Refs. [25, 26]) for rather different reasons. Since there are no long range forces between local monopoles, their number density in comoving coordinates does not decrease. Since their contribution to the energy density scales as $a^{-3}(t)$, they will come to dominate the mass of the Universe, provided $\eta$ is sufficiently large.

Theories with global monopoles [32, 33] are not ruled out, since there are long range forces between monopoles which lead to a "scaling solution" with a fixed number of monopoles per Hubble volume.

Let us now briefly discuss some of the basic mechanisms of the cosmic string scenario of structure formation. The implementation of the mechanisms in concrete models will be covered in the following sections.

The starting point of the structure formation scenario in the cosmic string theory is the scaling solution for the cosmic string network, according to which at all times $t$ (in particular at $t_{e q}$, the time when perturbations can start to grow) there will be a few long strings crossing each Hubble volume, plus a distribution of loops of radius $R \ll t$.

The cosmic string model admits three mechanisms for structure formation: loops, filaments, and wakes. Cosmic string loops have the same time averaged field as a point source with mass [34]

$$
M(R)=\beta R \mu,
$$

$R$ being the loop radius and $\beta \sim 2 \pi$. Hence, loops will be seeds for spherical accretion of dust and radiation.

For loops with $R \leq t_{e q}$, growth of perturbations in a model dominated by cold dark matter starts at $t_{e q}$. Hence, the mass at the present time will be

$$
M\left(R, t_{0}\right)=z\left(t_{e q}\right) \beta R \mu .
$$

In the original cosmic string model $[16,35]$ it was assumed that loops dominate over wakes. In this case, the theory could be normalized (i.e., $\mu$ could be determined) by demanding that loops with the mean separation of clusters $d_{c l}$ accrete the correct mass, i.e., that

$$
M\left(R\left(d_{c l}\right), t_{0}\right)=10^{14} M_{\odot} .
$$

This condition yields [35]

$$
\mu \simeq 10^{32} \mathrm{GeV}^{2}
$$

Thus, if cosmic strings are to be relevant for structure formation, they must arise due to a symmetry breaking at an energy scale $\eta \simeq 10^{16} \mathrm{GeV}$. This scale happens to be the scale of unification (GUT) of weak, strong and electromagnetic interactions. It is tantalizing to speculate that cosmology is telling us that there indeed was new physics at the GUT scale. 
The second mechanism involves long strings moving with relativistic speed in their normal plane which give rise to velocity perturbations in their wake [36]. The mechanism is illustrated in Fig. 1: space normal to the string is a cone with deficit angle [37]

$$
\alpha=8 \pi G \mu \text {. }
$$

If the string is moving with normal velocity $v$ through a bath of dark matter, a velocity perturbation

$$
\delta v=4 \pi G \mu v \gamma
$$

[with $\gamma=\left(1-v^{2}\right)^{-1 / 2}$ ] towards the plane behind the string results. At times after $t_{e q}$, this induces planar over-densities, the most prominent (i.e., thickest at the present time) and numerous of which were created at $t_{e q}$, the time of equal matter and radiation [38, 39, 40]. The corresponding planar dimensions are (in comoving coordinates)

$$
t_{e q} z\left(t_{e q}\right) \times t_{e q} z\left(t_{e q}\right) v \sim(40 \times 40 v) \mathrm{Mpc}^{2} .
$$

The thickness $d$ of these wakes can be calculated using the Zel'dovich approximation [40]. The result is

$$
d \simeq G \mu v \gamma(v) z\left(t_{e q}\right)^{2} t_{e q} \simeq 4 v \mathrm{Mpc} .
$$

Wakes arise if there is little small scale structure on the string. In this case, the string tension equals the mass density, the string moves at relativistic speeds, and there is no local gravitational attraction towards the string.

In contrast, if there is small scale structure on strings, then the string tension $T$ is smaller [41] than the mass per unit length $\mu$ and the metric of a string in $z$ direction becomes [42]

$$
d s^{2}=\left(1+h_{00}\right)\left(d t^{2}-d z^{2}-d r^{2}-(1-8 G \mu) r^{2} d y^{2}\right)
$$

with

$$
h_{00}=4 G(\mu-T) \ln \frac{r}{r_{0}},
$$

$r_{0}$ being the string width. Since $h_{00}$ does not vanish, there is a gravitational force towards the string which gives rise to cylindrical accretion, thus producing filaments.

As is evident from the last term in the metric (43), space perpendicular to the string remains conical, with deficit angle given by (39). However, since the string is no longer relativistic, the transverse velocities $v$ of the string network are expected to be smaller, and hence the induced wakes will be shorter and thinner. 
Which of the mechanisms - filaments or wakes - dominates is determined by the competition between the velocity induced by $h_{00}$ and the velocity perturbation of the wake. The total velocity is [42]

$$
u=-\frac{2 \pi G(\mu-T)}{v \gamma(v)}-4 \pi G \mu v \gamma(v),
$$

the first term giving filaments, the second producing wakes. Hence, for small $v$ the former will dominate, for large $v$ the latter.

By the same argument as for wakes, the most numerous and prominent filaments will have the distinguished scale

$$
t_{e q} z\left(t_{e q}\right) \times d_{f} \times d_{f}
$$

where $d_{f}$ can be calculated using the Zel'dovich approximation [43].

The cosmic string model predicts a scale-invariant spectrum of density perturbations, exactly like inflationary Universe models but for a rather different reason. Consider the r.m.s. mass fluctuations on a length scale $2 \pi k^{-1}$ at the time $t_{H}(k)$ when this scale enters the Hubble radius $H^{-1}(t)$. From the cosmic string scaling solution it follows that a fixed (i.e., $t_{H}(k)$ independent) number $\tilde{v}$ of strings of length of the order $t_{H}(k)$ contribute to the mass excess $\delta M\left(k, t_{H}(k)\right)$. Thus

$$
\frac{\delta M}{M}\left(k, t_{H}(k)\right) \sim \frac{\tilde{v} \mu t_{H}(k)}{G^{-1} t_{H}^{-2}(k) t_{H}^{3}(k)} \sim \tilde{v} G \mu .
$$

Note that the above argument predicting a scale invariant spectrum will hold for all topological defect models which have a scaling solution, in particular also for global monopoles and textures.

The amplitude of the r.m.s. mass fluctuations (equivalently: of the power spectrum) can be used to normalize $G \mu$. Since today on galaxy cluster scales

$$
\frac{\delta M}{M}\left(k, t_{0}\right) \sim 1,
$$

the growth rate of fluctuations linear in $a(t)$ yields

$$
\frac{\delta M}{M}\left(k, t_{e q}\right) \sim 10^{-4},
$$

and therefore, using $\tilde{v} \sim 10$,

$$
G \mu \sim 10^{-5}
$$

In contrast to the situation in inflationary Universe models, hot dark matter (HDM) is not from the outset ruled out as a dark matter candidate. 
As non-adiabatic seeds, cosmic string loops survive free streaming and can generate nonlinear structures on galactic scales, as discussed in detail in Refs. [44, 45]. Accretion of hot dark matter by a string wake was studied in Ref. [40]. In this case, nonlinear perturbations develop only late. At some time $t_{n l}$, all scales up to a distance $q_{\max }$ from the wake center go nonlinear. Here

$$
q_{\max } \sim G \mu v \gamma(v) z\left(t_{e q}\right)^{2} t_{e q} \sim 4 v \mathrm{Mpc},
$$

is the comoving thickness of the wake at $t_{n l}$. Demanding that $t_{n l}$ corresponds to a redshift greater than 1 leads to the constraint

$$
G \mu>5 \cdot 10^{-7} \text {. }
$$

Note that in a cosmic string and hot dark matter model, wakes form nonlinear structures only very recently. Accretion onto loops [46] and onto filaments [47] provide two mechanisms which may lead to high redshift objects such as quasars and high redshift galaxies.

\section{Introduction to high precision calculations with defects}

The last decade has witnessed unprecedented progress in mapping the cosmic microwave background (CMB) temperature anisotropy and the large scale structure (LSS) of the Universe. The prospect of fast improving data has forced theorists to new standards of precision in computing observable quantities. The new standards have been met in theories based on cosmic inflation[48, 49]. Topological defect scenarios [3, 4] have been more challenging. However, recently there have been a number of computational breakthroughs in defect theories, partly related to improvements in computer technology. Most strikingly, the method described in [50] showed how one could glean from defect simulations all the information required to compute accurately CMB and LSS power spectra. In this method the simulations are used uniquely for evaluating the two point functions (known as unequal time correlators, or UETCs) of the defects' stress-energy tensor. UETCs are all that is required for computing CMB and LSS power spectra. Furthermore, they are constrained by requirements of self-similarity (or scaling) and causality, which enable us to radically extend the dynamical range of simulations, a fact central to the success of the method.

This method was applied to theories based on global symmetries. In recent work $[51,52]$ we have shown how the same method could be applied to local cosmic strings (see also $[53,54]$ ). In the next few Sections we shall describe in detail the simulation and measurement of UETCs which led to the work in [51, 52]; as well as present the analytical tools used in this enterprise. The formalism we had to use is unfortunately more complicated than [50]. Local strings have an extra complication over global defects, which 
stems from the fact that we are unable to simulate the underlying field theory. Instead, we approximate the true dynamics with line-like relativistic strings. This is thought to be reasonable for the large scale properties of the stress-energy tensor, but we do not have a good understanding of how the string network loses energy in order to maintain scaling.

This leads to two problems. Firstly one is forced to make assumptions about which cosmological fluids pick up this deficit. It is often assumed that all the strings' energy and momentum is radiated into gravitational waves, approximated by a relativistic fluid. This is by no means certain, and it may well be that the energy and momentum is transferred to particles [55], and hence to the baryon, photon and CDM components.

Secondly, it is not enough to find correlators for a reduced number of stress energy tensor components (two scalar, two vector, two tensor), and then find the others by means of energy conservation. If energy conservation can be used then one needs to compute 3 scalar, 1 vector, and 1 tensor UETCs. If one is not allowed to make use of energy conservation, one has to compute 10 scalar, 3 vector, and 1 tensor UETC.

In the following sections we first give a qualitative description of the technical novelties introduced in defect scenarios. We describe defects as active incoherent perturbations. We then describe a set of tools with which we can perform high accuracy calculations of structure power spectra in these scenarios.

\section{Defects as active, incoherent perturbations}

We first focus on the basic assumptions of inflationary and defect theories and isolate the most striking contrasting properties. We define the concepts of active and passive perturbations, and of coherent and incoherent perturbations. In terms of these concepts inflationary perturbations are passive coherent perturbations. Defect perturbations are active perturbations more or less incoherent depending on the defect [56].

\subsection{ACTIVE AND PASSIVE PERTURBATIONS, AND THEIR DIFFERENT PERCEPTIONS OF CAUSALITY AND SCALING}

The way in which inflationary and defect perturbations come about is radically different. Inflationary fluctuations were produced at a remote epoch, and were driven far outside the Hubble radius by inflation. The evolution of these fluctuations is linear (until gravitational collapse becomes non-linear at late times), and we call these fluctuations "passive". Also, because all scales observed today have been in causal contact since the onset of inflation, causality does not strongly constrain the fluctuations which result. In contrast, defect fluctuations are continuously seeded by defect evolution, 
which is a non-linear process. We therefore say these are "active" perturbations. Also, the constraints imposed by causality on defect formation and evolution are much greater than those placed on inflationary perturbations.

\subsubsection{Active and passive scaling}

The notion of scale invariance has different implications in these two types of theory. For instance, a scale invariant gauge-invariant potential $\Phi$ with dimensions $L^{3 / 2}$ has a power spectrum

$$
P(\Phi)=\left\langle\left|\Phi_{\mathbf{k}}\right|^{2}\right\rangle \propto k^{-3}
$$

in passive theories (the Harrison-Zel'dovich spectrum). This results from the fact that the only variable available is $k$, and so the only spectrum one can write down which has the right dimensions and does not have a scale is the Harrison-Zel'dovich spectrum. The situation is different for active theories, since time is now a variable. The most general counterpart to the Harrison-Zel'dovich spectrum is

$$
P(\Phi)=\eta^{3} F_{\Phi}(k \eta)
$$

where $F_{\Phi}$ is, to begin with, an arbitrary function of $x=k \eta$. All other variables may be written as a product of a power of $\eta$, ensuring the right dimensions, and an arbitrary function of $x$. Inspecting all equations it can be checked that it is possible to do this consistently for all variables. All equations respect scaling in the active sense.

\subsubsection{Causality constraints on active perturbations}

Moreover, active perturbations are constrained by causality, in the form of integral constraints [57, 58]. These consist of energy and momentum conservation laws for fluctuations in an expanding Universe. The integral constraints can be used to find the low $k$ behaviour of the power spectrum of the perturbations, assuming their causal generation and evolution [59]. Typically, it is found that the causal creation and evolution of defects requires that their energy $\rho^{s}$ and scalar velocity $v^{s}$ be white noise at low $k$, but that the total energy power spectrum of the fluctuations is required to go like $k^{4}$. To reconcile these two facts one is forced to consider the compensation. This is an under-density in the matter-radiation energy density with a white noise low $k$ tail, correlated with the defect network so as to cancel the defects' white-noise tail. When one combines the defects energy with the compensation density, one finds that the gravitational potentials they generate also have to be white noise at large scales [56]. Typically the scaling function $F_{\Phi}(k \eta)$ will start as a constant and decay as a power law for $x=k \eta>x_{c}$. The value $x_{c}$ is a sort of coherence wavenumber of the defect. 
The larger it is the smaller the defect is. For instance $x_{c} \approx 12$ for cosmic strings (thin, tiny objects), whereas $x_{c} \approx 5.5$ for textures (round, fat, big things). Sophisticated work on causality [58] has shed light on how small $x_{c}$ may be before violating causality. The limiting lower bound $x_{c} \approx 2.7$ has been suggested.

Although we will not here have a chance to dwell on technicalities, it should be stated that the rather general discussion presented above is enough to determine the general form of the potentials for active perturbations. This has been here encoded in the single parameter $x_{c}$. We shall see that $x_{c}$ will determine the Doppler peak position for active perturbations. Doppler peaks are driven by the gravitational potential, so it should not be surprising that the defect length scale propagates into its potential, and from that into the position of the Doppler peaks.

\subsection{COHERENT AND INCOHERENT PERTURBATIONS}

Active perturbations may also differ from inflation in the way "chance" comes into the theory. Randomness occurs in inflation only when the initial conditions are set up. Time evolution is linear and deterministic, and may be found by evolving all variables from an initial value equal to the square root of their initial variances. By squaring the result one obtains the variances of the variables at any time. Formally, this results from unequal time correlators of the form

$$
\left\langle\Phi(\mathbf{k}, \eta) \Phi\left(\mathbf{k}^{\prime}, \eta^{\prime}\right)\right\rangle=\delta\left(\mathbf{k}-\mathbf{k}^{\prime}\right) \sigma(\Phi(k, \eta)) \sigma\left(\Phi\left(k, \eta^{\prime}\right)\right),
$$

where $\sigma$ denotes the square root of the power spectrum $P$. In defect models however, randomness may intervene in the time evolution as well as the initial conditions. Although deterministic in principle, the defect network evolves as a result of a complicated non-linear process. If there is strong non-linearity, a given mode will be "driven" by interactions with the other modes in a way which will force all different-time correlators to zero on a time scale characterized by the "coherence time" $\theta_{c}(k, \eta)$. Physically this means that one has to perform a new "random" draw after each coherence time in order to construct a defect history [56]. The counterpart to (54) for incoherent perturbations is

$$
\left\langle\Phi(\mathbf{k}, \eta) \Phi\left(\mathbf{k}^{\prime}, \eta^{\prime}\right)\right\rangle=\delta\left(\mathbf{k}-\mathbf{k}^{\prime}\right) P\left(\Phi(k, \eta), \eta^{\prime}-\eta\right) .
$$

For $\left|\eta^{\prime}-\eta\right| \equiv|\Delta \eta|>\theta_{c}(k, \eta)$ we have $P(\Phi(k, \eta), \Delta \eta)=0$. For $\Delta \eta=0$, we recover the power spectrum $P(\Phi(k, \eta), 0)=P(\Phi(k, \eta))$.

We shall label as coherent and incoherent (54) and (55) respectively. This feature does not affect the position of the Doppler peaks, but it does affect the structure of secondary oscillations. An incoherent potential will 
drive the CMB oscillator incoherently, and therefore it may happen that the secondary oscillations get washed out as a result of incoherence.

\section{Tool 1: Scalar, vector, and tensor decomposition}

Having identified the main qualitative novelties in defecy calculations, we now proceed to present the set of tools required for performing high accuracy calculations in these scenarios. We start with the decomposition into scalar, vector, and tensor components. Let $\Theta_{\mu \nu}(\mathbf{x})$ be the defect stressenergy tensor. We may Fourier analyze it

$$
\Theta_{\mu \nu}(\mathbf{x})=\int d^{3} k \Theta_{\mu \nu}(\mathbf{k}) e^{i \mathbf{k} \cdot \mathbf{x}}
$$

and decompose its Fourier components as:

$$
\begin{aligned}
\Theta_{00}= & \rho^{d} \\
\Theta_{0 i}= & i \hat{k}_{i} v^{d}+\omega_{i}^{d} \\
\Theta_{i j}= & p^{d} \delta_{i j}+\left(\hat{k}_{i} \hat{k}_{j}-\frac{1}{3} \delta_{i j}\right) \Pi^{S}+ \\
& i\left(\hat{k}_{i} \Pi_{j}^{V}+\hat{k}_{j} \Pi_{i}^{V}\right)+\Pi_{i j}^{T}
\end{aligned}
$$

with $\hat{k}^{i} \omega_{i}^{d}=0, \hat{k}^{i} \Pi_{i}^{V}=0, \hat{k}^{i} \Pi_{i j}^{T}=0$, and $\Pi_{i}^{T i}=0$. The variables $\left\{\rho^{d}, v^{d}, p^{d}, \Pi^{S}\right\}$ are the scalars, $\left\{\omega_{i}^{d}, \Pi_{i}^{V}\right\}$ the vectors, and $\Pi_{i j}^{T}$ the tensors. The decomposition can be inverted by means of

$$
\begin{aligned}
v^{d} & =-i \hat{k}^{i} \Theta_{0 i} \\
\omega_{i}^{d} & =\left(\delta_{i}^{j}-\hat{k}^{i} \hat{k}_{j}\right) \Theta_{0 j}
\end{aligned}
$$

and

$$
\begin{aligned}
p^{s}= & \frac{1}{3} \Theta_{i}^{i} \\
\Pi^{S}= & \frac{3}{2}\left(\hat{k}^{i} \hat{k}^{j}-\frac{1}{3} \delta^{i j}\right) \Theta_{i j} \\
\Pi_{i}^{V}= & -i\left(\hat{k}^{j} \delta_{i}^{l}-\hat{k}_{i} \hat{k}^{j} \hat{k}^{l}\right) \Theta_{l j} \\
\Pi_{i j}^{T}= & \left(\delta_{i}^{l} \delta_{j}^{m}-\frac{1}{2} \delta_{i j} \delta^{l m}+\frac{1}{2} \hat{k}_{i} \hat{k}_{j} \hat{k}^{l} \hat{k}^{m}+\frac{1}{2}\left(\hat{k}^{l} \hat{k}^{m} \delta_{i j}+\right.\right. \\
& \left.\left.\hat{k}_{i} \hat{k}_{j} \delta^{l m}\right)-\left(\hat{k}_{i} \hat{k}^{l} \delta_{j}^{m}+\hat{k}_{j} \hat{k}^{l} \delta_{i}^{m}\right)\right) \Theta_{l m}
\end{aligned}
$$

although simpler recursive formulae may be written.

This decomposition and choice of harmonics is slightly different from the one used in Hu and White [49], and Kodama and Sasaki [60]. The reason for this is that in [49] and [60] one assumes that all variables represent 
square roots of power spectra. Isotropy can then be assumed. This procedure will indeed lead to the right ensemble average $P(k)$ and $C_{\ell}$ if the source is coherent (or a decomposition into coherent eigenmodes has been performed.) However it is not valid for each realization of an incoherent source.

\section{Tool 2: Unequal-time correlators}

The unequal time correlators are defined as

$$
\left\langle\Theta_{\mu \nu}(\mathbf{k}, \eta) \Theta_{\alpha \beta}^{\star}\left(\mathbf{k}, \eta^{\prime}\right)\right\rangle \equiv \mathcal{C}_{\mu \nu, \alpha \beta}\left(k, \eta, \eta^{\prime}\right)
$$

where $\Theta_{\mu \nu}$ is the stress energy tensor, $\mathbf{k}$ is the wave-vector, and $\eta$ and $\eta^{\prime}$ are any two (conformal) times. The UETCs determine all other 2 point functions, most notably CMB and LSS power spectra $C_{\ell}$ and $P(k)$.

All correlators between modes at $(\mathbf{k}, \eta)$ and $\left(\mathbf{k}^{\prime}, \eta^{\prime}\right)$ will be proportional to $\delta\left(\mathbf{k}-\mathbf{k}^{\prime}\right)$ due to translational invariance. We shall drop this factor in all formulae. The correlators can also be functions of $k$ alone, due to isotropy. Since conjugation corresponds to $\mathbf{k} \rightarrow-\mathbf{k}$, isotropy implies that the correlators must be real. Because of incoherence the correlators will be generic functions of $\eta$ and $\eta^{\prime}$.

Furthermore, the form of the $\mathrm{S}+\mathrm{V}+\mathrm{T}$ decomposition fixes further the form of the correlators. One can always write down the most general form of a correlator, and then contract the result with $\hat{k}_{i}$ or $\delta_{i j}$, wherever appropriate, to obtain further conditions. For instance for any scalar $S$ and vector $V_{i}$ we could write

$$
\left\langle S V_{i}\right\rangle=\alpha(k) \hat{k}_{i}
$$

By contracting with $\hat{k}_{i}$ we would then conclude that $\alpha(k)=0$. Proceeding

in this way we can show that cross correlators involving components of different type (S, V, or T) must be zero. Furthermore for any two vectors $V_{i}, W_{i}$, one has

$$
\left\langle V_{i} W_{j}^{*}\right\rangle=f^{V W}\left(k, \eta, \eta^{\prime}\right)\left(\delta_{i j}-\hat{k}_{i} \hat{k}_{j}\right)
$$

and so instead of 6 functions we have only one. In order to agree with conventions used in the literature we shall instead define this single function by means of

$$
f^{V W}\left(k, \eta, \eta^{\prime}\right)=\sum_{i}\left\langle V_{i} W_{i}^{*}\right\rangle
$$

For correlators involving two tensor quantities one can use similar arguments to prove the general form

$$
\begin{aligned}
\left\langle\Pi_{i j}^{T} \Pi_{k l}^{T \star}\right\rangle= & f^{\Pi^{T} \Pi^{T}}\left(k, \eta, \eta^{\prime}\right)\left(\delta_{i j} \delta_{k l}-\left(\delta_{i k} \delta_{j l}+\delta_{i l} \delta_{j k}\right)\right. \\
& -\left(\delta_{i j} \hat{k}_{k} \hat{k}_{l}+\delta_{k l} \hat{k}_{i} \hat{k}_{j}\right)+\left(\delta_{i k} \hat{k}_{j} \hat{k}_{l}+\delta_{i l} \hat{k}_{j} \hat{k}_{k}+\right. \\
& \left.\left.\delta_{j k} \hat{k}_{i} \hat{k}_{l}+\delta_{j l} \hat{k}_{i} \hat{k}_{k}\right)-\hat{k}_{i} \hat{k}_{j} \hat{k}_{k} \hat{k}_{l}\right)
\end{aligned}
$$


and again we have a single function rather than 21. Again in order to comply with conventions in the literature we shall define this single function by means of

$$
f^{\Pi^{T} \Pi^{T}}\left(k, \eta, \eta^{\prime}\right)=\sum_{i j}\left\langle\Pi_{i j}^{T} \Pi_{i j}^{T \star}\right\rangle
$$

Hence there should be 10 scalar correlator functions

$$
\begin{array}{cccc}
f^{\rho^{d} \rho^{d}} & f^{\rho^{d} v^{d}} & f^{\rho^{d} p^{d}} & f^{\rho^{d} \Pi^{S}} \\
\ldots & f^{v^{d} v^{d}} & f^{v^{d} p^{d}} & f^{v^{d} \Pi^{S}} \\
\ldots & \cdots & f^{p^{d} p^{d}} & f^{p^{d} \Pi^{S}} \\
\ldots & \ldots & \ldots & f^{\Pi^{S} \Pi^{S}}
\end{array}
$$

3 vector correlators:

$$
\begin{array}{cc}
f^{\omega^{d} \omega^{d}} & f^{\omega^{d} \Pi^{V}} \\
\cdots & f^{\Pi^{V} \Pi^{V}}
\end{array}
$$

and a single tensor correlator function $f^{\Pi^{T} \Pi^{T}}$.

In general these functions are functions of $\left(k, \eta, \eta^{\prime}\right)$, and this is indeed the case during the matter radiation transition. However well into the matter and radiation epochs there is scaling, and these functions may be written as:

$$
f^{X Y}\left(k, \eta, \eta^{\prime}\right)=\frac{F^{X Y}\left(x, x^{\prime}\right)}{\sqrt{\eta \eta^{\prime}}}
$$

where $X Y$ represents any pair of superscripts considered above, and $x=k \eta$ and $x^{\prime}=k \eta^{\prime}$. The above scaling form results from the dimensional analysis argument: $\left[\Theta_{\mu \nu}(x)\right]=1 / L^{2},\left[\Theta_{\mu \nu}(k)\right]=L,[\delta(\mathbf{k})]=L^{3},\left[f^{X Y}\right]=1 / L$. We have used units where $G=c=1$.

\section{Causality constraints}

From analyticity conditions [61] we can use isotropy and symmetry [61, 62] to derive general expansions in $\mathbf{k}$ around $k_{i}=0$. This is usually very helpful to check numerical results. We find:

$$
\begin{aligned}
\left\langle\Theta_{00}(k, \eta) \Theta_{00}\left(k, \eta^{\prime}\right)\right\rangle= & X \\
\left\langle\Theta_{00}(k, \eta) \Theta_{0 i}\left(k, \eta^{\prime}\right)\right\rangle= & i k_{i} Y \\
\left\langle\Theta_{00}(k, \eta) \Theta_{i j}\left(k, \eta^{\prime}\right)\right\rangle= & V \delta_{i j}+W k_{i} k_{j} \\
\left\langle\Theta_{0 i}(k, \eta) \Theta_{0 j}\left(k, \eta^{\prime}\right)\right\rangle= & T \delta_{i j}+U k_{i} k_{j} \\
\left\langle\Theta_{0 i}(k, \eta) \Theta_{j k}\left(k, \eta^{\prime}\right)\right\rangle= & i\left[Q k_{i} \delta_{j k}+R\left(k_{j} \delta i k+k_{k} \delta_{i j}\right)\right. \\
& \left.+S k_{i} k_{j} k_{k}\right]
\end{aligned}
$$




$$
\begin{aligned}
\left\langle\Theta_{i j}(k, \eta) \Theta_{k l}\left(k, \eta^{\prime}\right)\right\rangle= & A \delta_{i j} \delta_{k l}+B\left(\delta_{i k} \delta_{j l}+\delta i l \delta j k\right) \\
& +C\left(k_{i} k_{j} \delta k l+k_{k} k_{l} \delta i j\right) \\
& +D\left(k_{i} k_{k} \delta j l+k_{i} k_{l} \delta j k+k_{j} k_{l} \delta i k\right. \\
& \left.+k_{j} k_{k} \delta i l\right)+E k_{i} k_{j} k_{k} k_{l}
\end{aligned}
$$

where $\mathrm{A}, \mathrm{B}$ etc. are functions independent of $k$. By applying the scalar, vector and tensor decomposition we can then find the specific form of the correlators in our formalism to obtain relations between the correlators

$$
\begin{aligned}
f^{\rho \rho} & =X \\
f^{\rho p} & =V+\frac{1}{3} k^{2} W \\
f^{\rho v} & =k Y \\
f^{\rho \Pi^{S}} & =\frac{3}{2} W\left(k^{2}-\frac{1}{3}\right) \\
f^{p p} & =A+\frac{2}{3} B+\frac{2}{3} k^{2}\left(C+\frac{2}{3} D\right)+\frac{1}{9} k^{4} E \\
f^{p v} & =k\left(Q+\frac{2}{3} R\right)+\frac{1}{3} S k^{2} \\
f^{p \Pi^{S}} & =\left(C-\frac{4}{3} D\right) k^{2}+\frac{1}{3} E k^{4} \\
f^{v v} & =-\left(T+k^{2} U\right) \\
f^{v \Pi^{S}} & =k\left(2 R-S k^{2}\right) \\
f^{\Pi^{S} \Pi^{S}} & =3 B+4 D k^{2}+E k^{4} \\
f^{\omega \omega} & =2 T \\
f^{\omega \Pi^{S}} & =2 R k \\
f^{\Pi^{V} \Pi^{V}} & =2 B \\
f^{\Pi^{T} \Pi^{T}} & =4 B
\end{aligned}
$$

We can now derive $\mathbf{k} \rightarrow 0$ constraints on the correlators. These may help to complete the functions $F^{X Y}$ near the origin, where they may not be accessible from simulations due to low sampling of points on the lattice. Specifically, we get ratios between the scalar, vector and tensor anisotropic stresses and between the momentum and vorticity,

$$
\begin{aligned}
f^{\Pi^{S} \Pi^{S}}: f^{\Pi^{V} \Pi^{V}}: f^{\Pi^{T} \Pi^{T}} & =3: 2: 4 \\
f^{v v}: f \omega \omega & =1: 2
\end{aligned}
$$

to zeroth order and,

$$
f^{v \Pi^{S}} \approx f^{\omega_{i} \Pi_{i}^{V}}
$$


to first order.

\section{Tool 3: Simulation determination of the correlators}

Having developped the necessary analytical tools we now describe how to apply them to simulations. We consider the work in [51]. The simulation starts with a network of strings obtained using the algorithm developed in [27] which simulates the symmetry breaking of the underlying field by selecting random phases for the field at each point in the lattice. Strings are identified on points in the lattice where the phase of the field has a nonzero winding number. The strings are described by their position $\mathbf{X}(\sigma, \eta)$ where $\sigma$ is a parameter running along the string and $\eta$ is conformal time. By imposing the following gauge conditions

$$
\dot{\mathbf{X}} \cdot \dot{\mathbf{X}}=0 \quad \dot{\mathbf{X}}^{2}+\dot{\mathbf{X}}^{2}=1
$$

where dots and primes denote derivatives with respect to $\eta$ and $\sigma$, respectively, the equation of motion takes on the simple form of the wave equation

$$
\ddot{\mathbf{X}}^{2}+\ddot{\mathbf{X}}^{2}=0
$$

and can be discretised on the lattice so that all the possible velocities take integer values only $[63,64]$. This greatly increases the accuracy and speed of string network codes based on this algorithm. The network is then evolved using the discretised equations of motion and intercommuting relations. To simulate the extraction of energy from the system due to the decay of the string loops into gravitational radiation and/or particles, loops of a minimum size are excised from the simulation at each time step. This also ensures that the network scales with respect to the conformal time $\eta$ which enables us to extend the dynamical range of the resulting correlation functions beyond the limited range covered in the simulation. Fig. 2 shows how the correlation length defined by $\xi=\sqrt{\mu / \rho_{l}}$ scales as a function of conformal time. Here, $\rho_{l}$ is the density of 'long' strings.

We performed simulations with box sizes ranging from $128^{3}$ to $600^{3}$, with a cut-off on the loop size of two links. Realisation averages were carried out with $256^{3}$ boxes once it was determined that the general form of the correlators scaled very accurately with box size. To evaluate the UETCs from the simulations we selected times in the range $0.1 N<t<N / 4$, where $N$ is the box size, when we were sure that the string network was scaling, and when boundary effects are still excluded by causality. At each

of the time steps the vectors $\dot{\mathbf{X}}$ and $\mathbf{X}$ from each point along the strings in the network were stored. From this we obtained the time evolution of the network's stress-energy tensor $\Theta_{\mu \nu}$ at each point on the lattice using

$$
\Theta_{\mu \nu}(\mathbf{x})=\mu \int d \sigma\left(\dot{X}^{\mu} \dot{X}^{\nu}-\dot{X}^{\mu} \dot{X}^{\nu}\right) \delta^{3}(\mathbf{x}-\mathbf{X}(\sigma, \eta))
$$




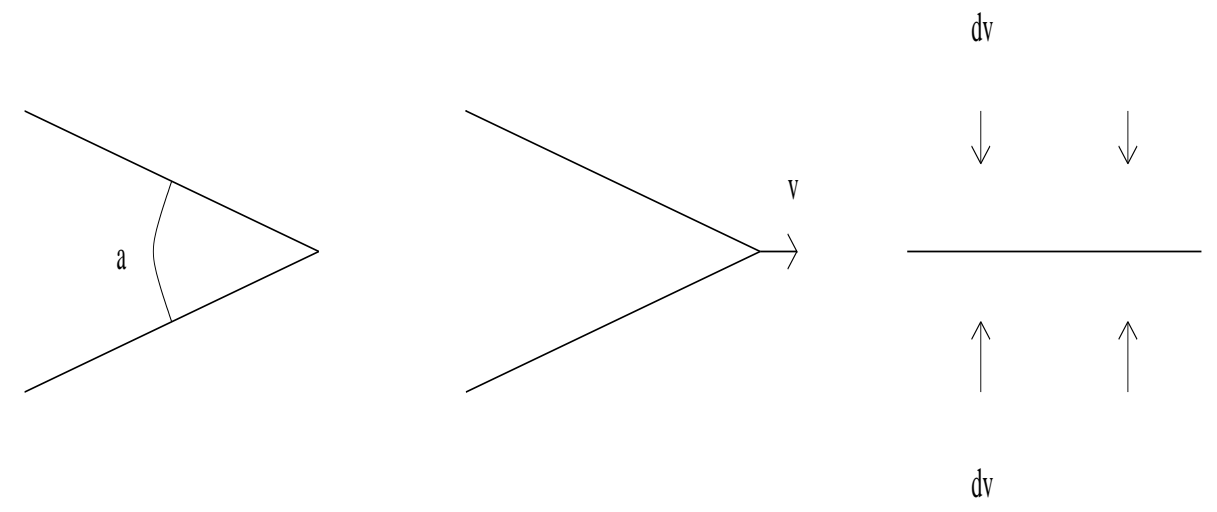

Figure 1. Sketch of the mechanism by which a long straight cosmic string $S$ moving with velocity $v$ in transverse direction through a plasma induces a velocity perturbation $\Delta v(d v)$ towards the wake. Shown on the left is the deficit angle $a$, in the center is a sketch of the string moving in the plasma, and on the right is the sketch of how the plasma moves towards the wake with velocity $d v$ in the frame in which the string is at rest.

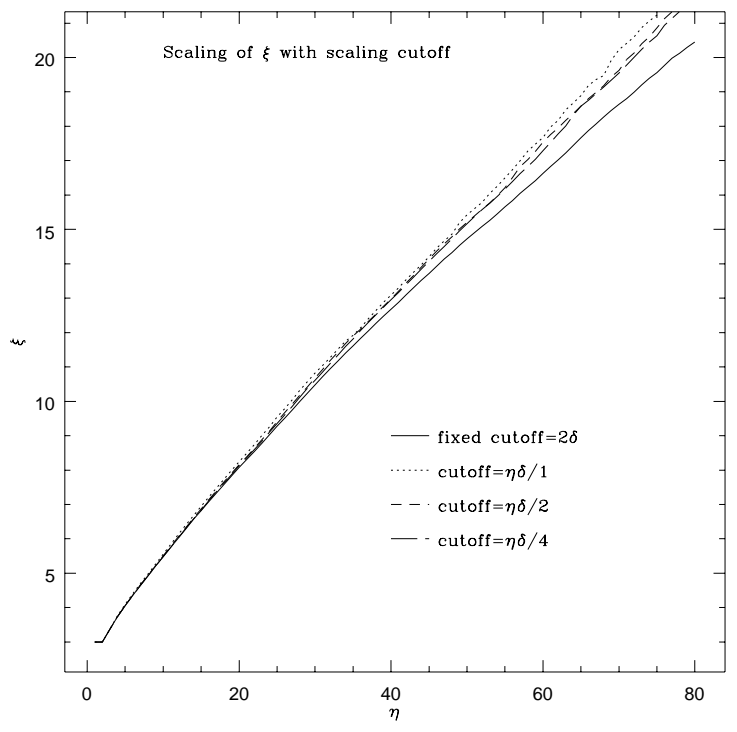

Figure 2. Scaling of the correlation length $\xi$ for various loop cut off sizes. 
The Fast Fourier Transforms of all 10 independent components of the string stress-energy were then decomposed into irreducible scalar, vector and tensor modes using eqns in Section (6). This resulted in all the SVT components being computed directly from the simulation without making assumptions on energy conservation and on the details of energy dissipation from the string network. The drawback of obtaining all the components directly in such a manner is that the process becomes computationally intensive for even modestly sized simulations, e.g. $256^{3}$, as, in effect, one has to deal with $\approx 10$ times the number of variables at each point on the lattice.

By cross-correlating the decomposed stress-energy components from a central time with those from all the stored time steps the 14 independent UETCs $f^{X Y}\left(k, \eta, \eta^{\prime}\right)$ were computed.

In Figs.3-16 we display the forms of the various functions $f^{X Y}(k, 25, \eta)$ where $k, \eta$ and $\eta^{\prime}$ are in lattice units. This is enough to infer the scaling functions $F^{X Y}\left(x, x^{\prime}\right)$. One can see that these correlators fall off very quickly away from the diagonal, a phenomenon known as incoherence [56] as we explained above. Incoherence determines whether or not we have enough dynamical range to compute the UETCs: if we see the fall off completly clearly we have enough dynamical range!

A striking feature of our results is the dominance of $\Theta_{00}$ over all other components (see Figs. 17 and 18). The string anisotropic stresses are in the predicted [61] ratios $\left|\Theta^{S}\right|^{2}:\left|\Theta^{V}\right|^{2}:\left|\Theta^{T}\right|^{2}$ of $3: 2: 4$, as $k \tau \rightarrow 0$. However $\left|\Theta_{00}\right|^{2} \gg\left|\Theta^{S}\right|^{2}$, and so scalars dominate over vectors and tensors. Also the energy density power spectrum rises from a white noise tail at $k \tau \approx 0$ into a peak at $k \tau \approx 20$, after which it falls off. Sub-horizon modes are therefore of great importance. These features consistently appeared for all box sizes, and are independent of the cutoff size imposed on the loops.

\section{Tool 4: Decomposition into eigenmodes}

The UETCs $c_{\mu \nu, \alpha \beta}\left(k \tau, k \tau^{\prime}\right)$ may be diagonalised [50] and written as

$$
c_{\mu \nu, \alpha \beta}\left(k \tau, k \tau^{\prime}\right)=\sum_{i} \lambda^{(i)} v_{\mu \nu}^{(i)}(k \tau) v_{\alpha \beta}^{(i)}\left(k \tau^{\prime}\right)
$$

where $\lambda^{(i)}$ are eigenvalues. In general, defects are incoherent sources for perturbations [56], which means that this matrix does not factorize into the product of two vectors $v_{\mu \nu}(k \tau) v_{\alpha \beta}\left(k \tau^{\prime}\right)$. Standard codes solving for CMB and LSS power spectra assume coherence. However we see that an incoherent source may be represented as an incoherent sum of coherent sources. We may therefore feed each eigenmode into standard codes [65] to 


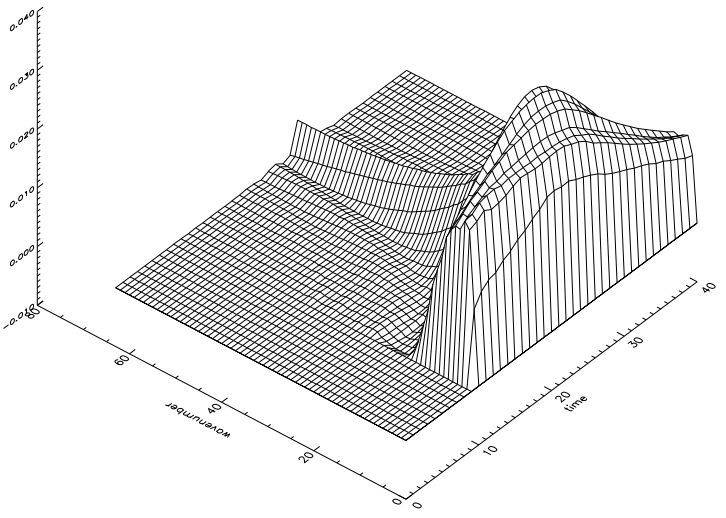

Figure 3. The function $\left\langle\left|\rho^{d}\right|^{2}\right\rangle(k, 25, \eta)$.

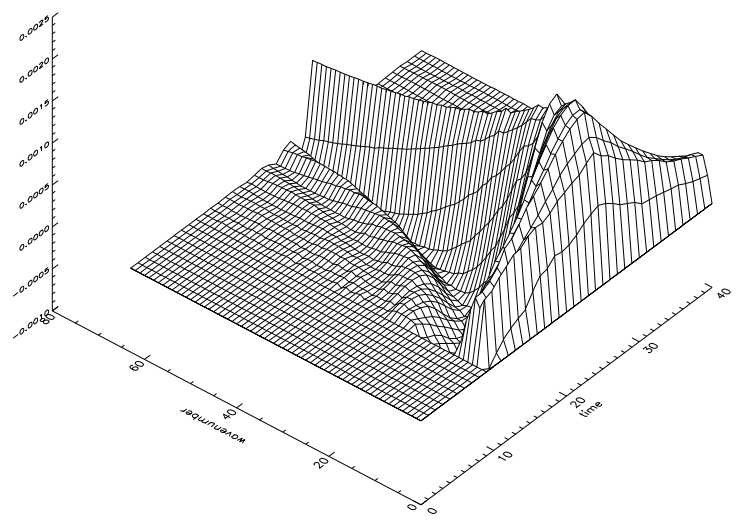

Figure 4. The function $\left\langle\left|v^{d}\right|^{2}\right\rangle(k, 25, \eta)$. 


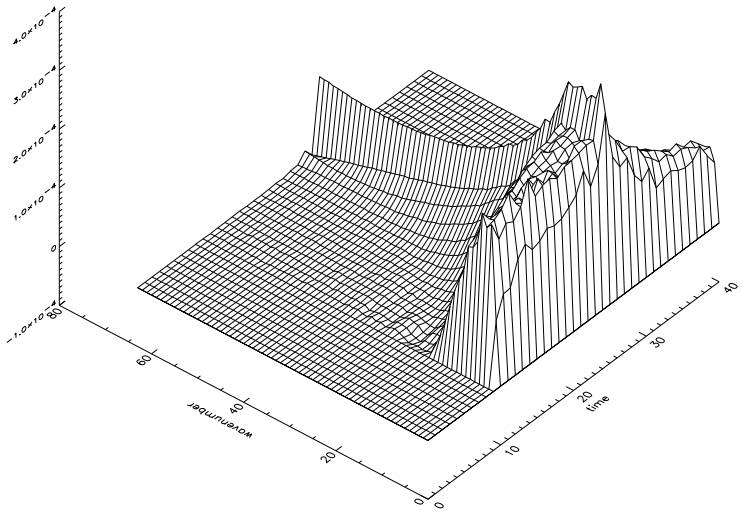

Figure 5. The function $\left\langle\left|p^{d}\right|^{2}\right\rangle(k, 25, \eta)$.

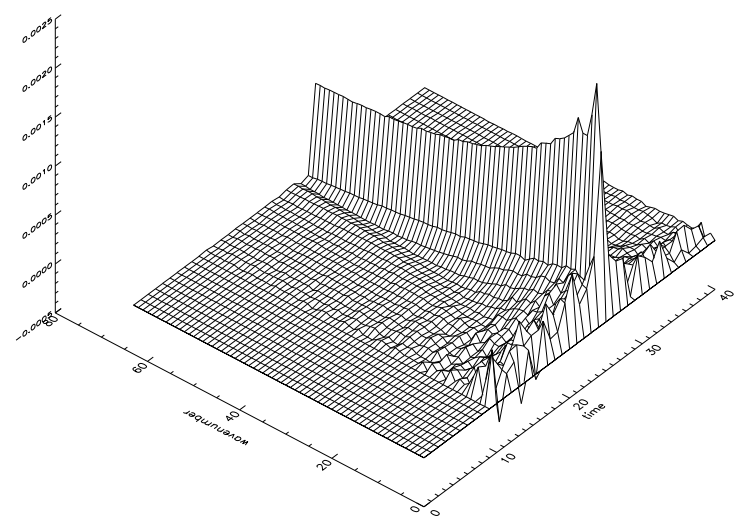

Figure 6. The function $\left\langle\left|\Pi^{S}\right|^{2}\right\rangle(k, 25, \eta)$. 


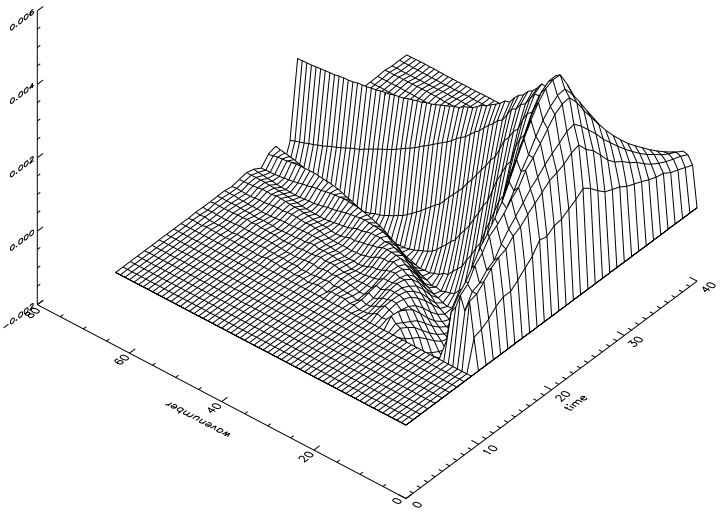

Figure 7. The function $\sum_{i}\left\langle\left|\omega_{i}^{d}\right|^{2}\right\rangle(k, 25, \eta)$.

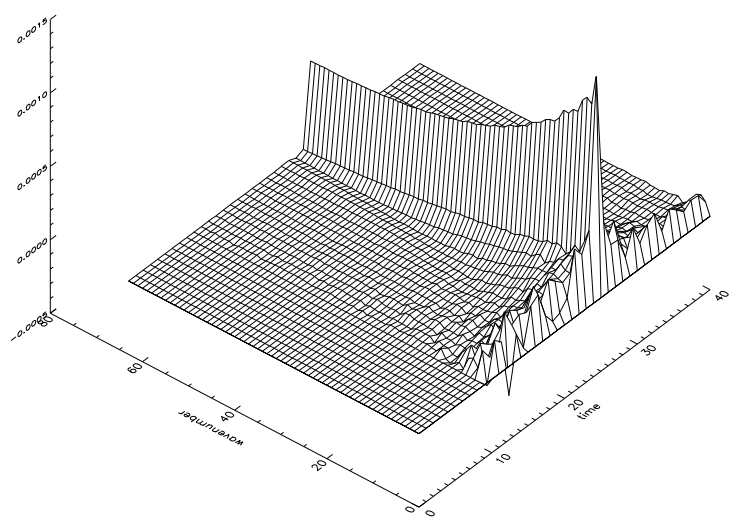

Figure 8. The function $\sum_{i}\left\langle\left|\Pi_{i}^{V}\right|^{2}\right\rangle(k, 25, \eta)$. 


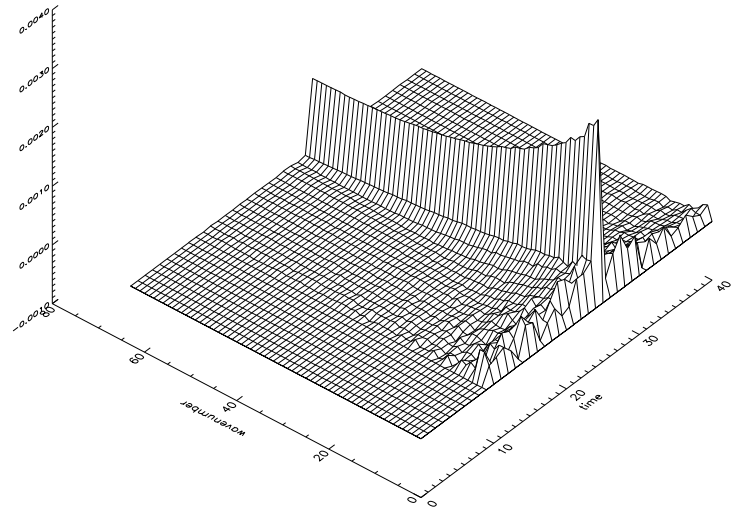

Figure 9. The function $\sum_{i j}\left\langle\left|\Pi_{i j}^{T}\right|^{2}\right\rangle(k, 25, \eta)$.

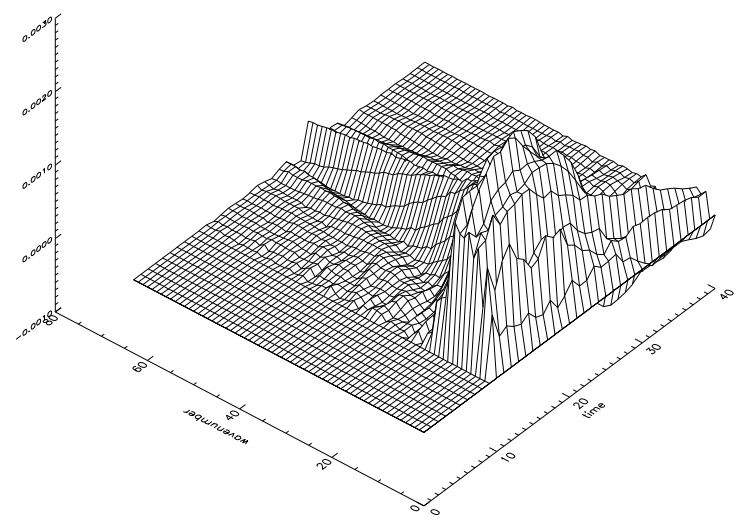

Figure 10. The function $\left\langle\rho^{d} v^{d \star}\right\rangle(k, 25, \eta)$. 


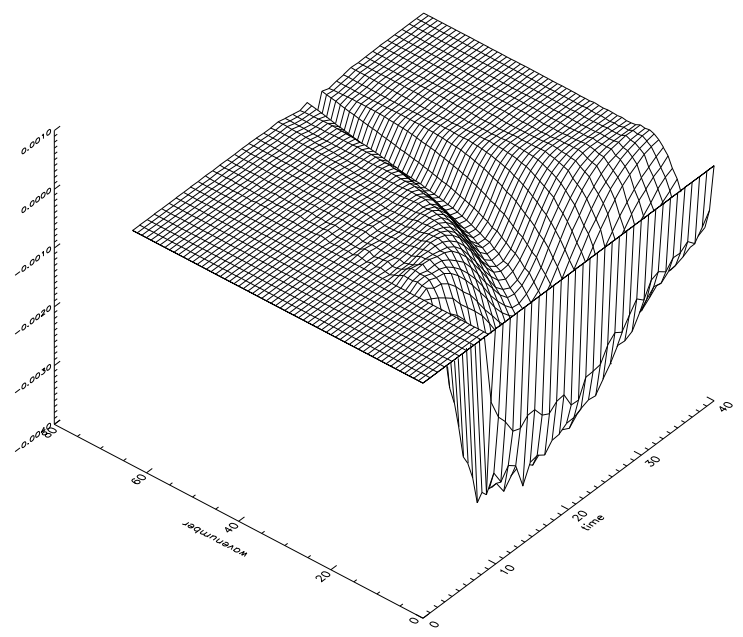

Figure 11. The function $\left\langle\rho^{d} p^{d \star}\right\rangle(k, 25, \eta)$.

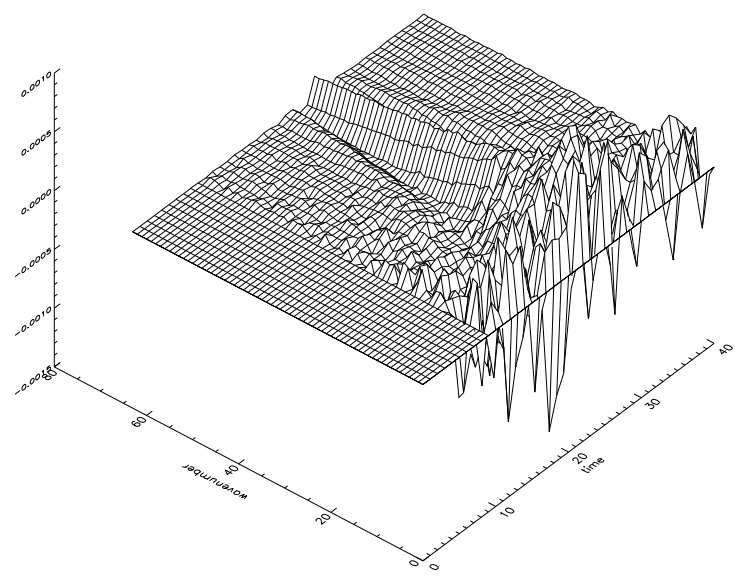

Figure 12. The function $\left\langle\rho^{d} \Pi^{S \star}\right\rangle(k, 25, \eta)$. 


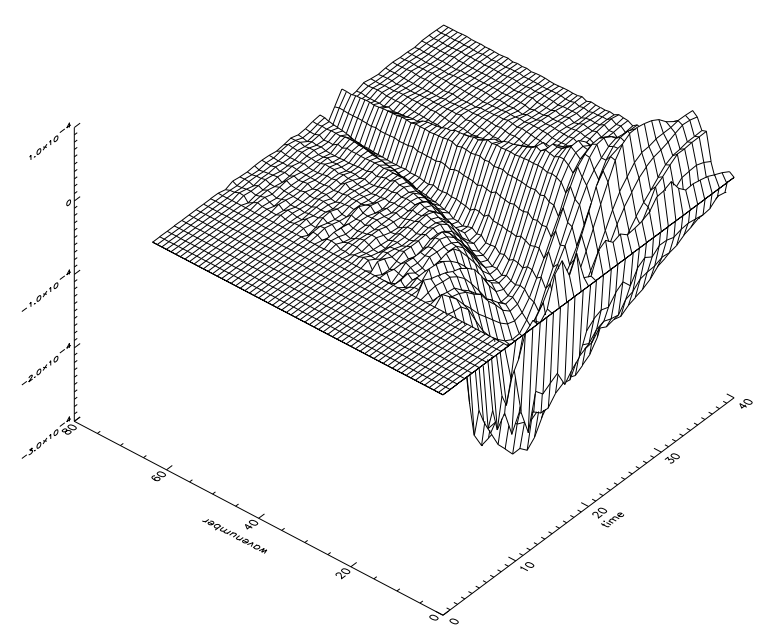

Figure 13. The function $\left\langle p^{d} v^{d \star}\right\rangle(k, 25, \eta)$.

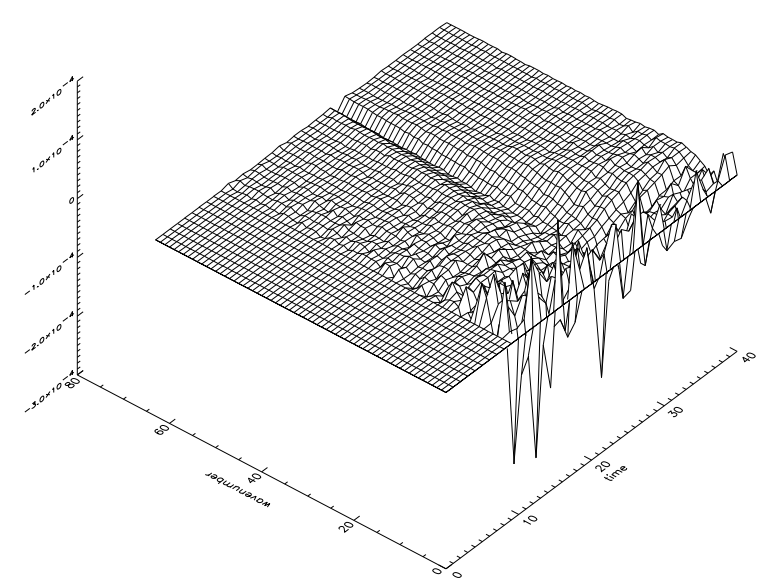

Figure 14. The function $\left\langle p^{d} \Pi^{S \star}\right\rangle(k, 25, \eta)$. 


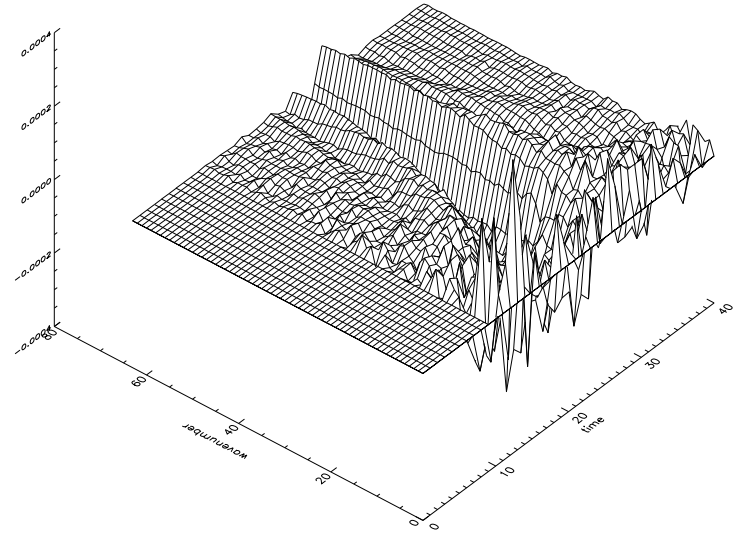

Figure 15. The function $\left\langle v^{d} \Pi^{S \star}\right\rangle(k, 25, \eta)$.

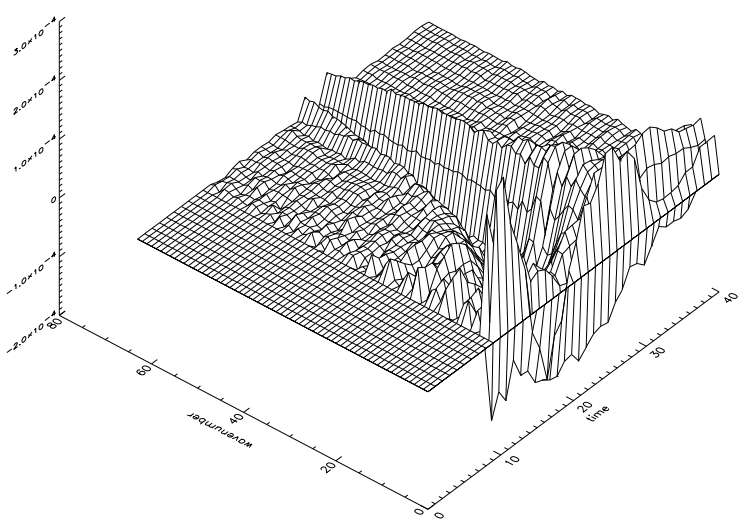

Figure 16. The function $\sum_{i}\left\langle\omega_{i}^{d} \Pi_{i}^{V \star}\right\rangle(k, 25, \eta)$. 


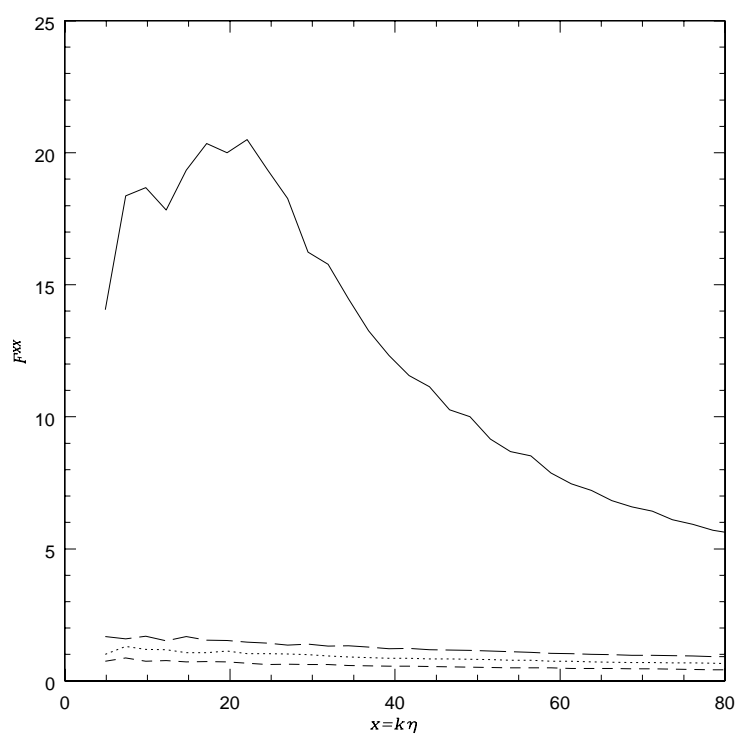

Figure 17. This plot shows the scaling autocorrelation of the energy density, the scalar vector and tensor anisotropic stresses. We see that the energy density dominates all the other components.

find the $C_{\ell}^{(i)}$ and $P^{(i)}(k)$ associated with each mode. The series $\sum \lambda^{(i)} C_{\ell}^{(i)}$ and $\sum \lambda^{(i)} P^{(i)}(k)$ provide convergent approximations to the power spectra.

In Fig. 19 we show the eigenmodes corresponding to the energy density $\Theta_{00}$. We see that the leading eigenmode carries the mark of the peak in the energy power spectrum at $x \approx 20$. The other eigenmodes become very small very quickly and change sign with higher and higher frequency. If we compute the $C_{\ell}$ power spectrum for each of these modes, and sum the series, we find quick convergence shortly after 20 modes have been included (see Fig. 20).

\section{Some results}

The methods described above supplied a variety of interesting results for cosmic strings. They show that local strings, unlike global defects have a Doppler peak. The main uncertainty seems to be in the details of how strings loose energy (see e.g. [66, 67]). String decay products are clearly the most uncertain aspect of cosmic string theory. By measuring the full 14 UETCs associated with long strings, we assume nothing about decay products when extracting information from simulations The simulations 


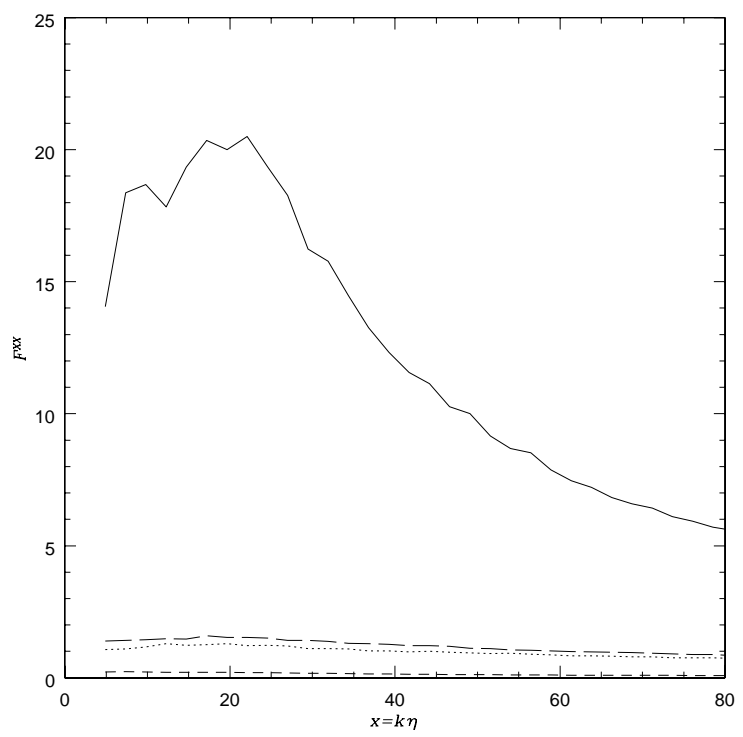

Figure 18. Same but for the energy density, the pressure, the velocity and the vorticity

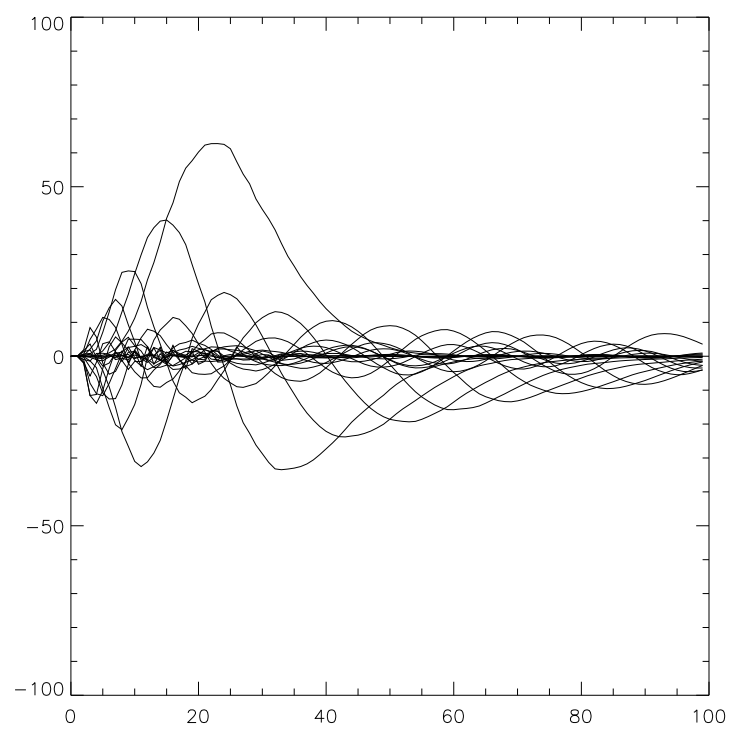

Figure 19. Decomposition into eigenmodes of the energy density. 
will then also place constraints upon the decay products.

In Fig. 21 we plot $\sqrt{ }\left[\ell(\ell+1) C_{\ell} / 2 \pi\right]$, setting the Hubble constant to $H_{0}=50 \mathrm{Km} \mathrm{sec}^{-1} \mathrm{Mpc}^{-1}$, the baryon fraction to $\Omega_{b}=0.05$, and assuming a flat geometry, no cosmological constant, 3 massless neutrinos, standard recombination, and cold dark matter. We also superimpose current experimental points. The most interesting feature is the presence of a reasonably high Doppler peak at $\ell=400-600$, following a pronouncedly tilted large angle plateau (cf. [68]). This feature sets local strings apart from global defects. It puts them in a better shape to face the current data.

The CMB power spectrum is relatively insensitive to the equation of state of the extra fluid. We have plotted results for $w^{X}=1 / 3,0.1,0.01$. Dumping some energy into CDM has negligible effect. Small dumps into baryon and radiation fluids, on the contrary, boost the Doppler peak very strongly. We plotted the effect of dumping $5 \%$ of the energy into the radiation fluid.

The LSS power spectra on the other hand is strongly dependent on $w^{X}$. In Fig. 22 we plotted the CDM power spectrum $P(k)$ together with experimental points as in [69]. The normalization has been fixed by COBE data points. We see that the peak of the spectrum is always at smaller scales than standard CDM predictions, or observations. However the overall normalization of the spectrum increases considerably as $w^{X}$ decreases.

The CDM rms fluctuation in $8 h^{-1} \mathrm{Mpc}$ spheres is $\sigma_{8}=0.4,0.6,1.8$ for $w^{X}=1 / 3,0.1,0.01$. Hence relativistic decay products match well the observed $\sigma_{8} \approx 0.5$. On the other hand in $100 h^{-1} \mathrm{Mpc}$ spheres one requires bias $b_{100}=\sigma_{100}^{\text {data }} / \sigma_{100}=4.9,3.7,1.6$ to match observations.

Energy dumps into radiation have no effect on the CDM power spectrum. However if there is energy transfer into CDM or baryons, even with $w^{X}=1 / 3$, the CDM power spectrum is highly enhanced. This is due to the addition of small scale entropy fluctuations to the usual fluctuations gravitationally induced by the stings. We plot the result of a $5 \%$ transfer into CDM and a $20 \%$ transfer into baryons (with $w^{X}=1 / 3$ ) for which $b_{100}=2.0,1.5$.

Hence in our calculations local strings have a bias problem at 100 $h^{-1} \mathrm{Mpc}$, although its magnitude is not as great as found in [54]. It depends sensitively on the decay products, being reduced if the strings have a channel into non-relativistic particles, or if there is some energy transfer into the baryon and CDM fluid. The main problem with strings in an $\Omega=1$, $\Omega_{b}=0.05, \Omega_{\Lambda}=0 \mathrm{CDM}$ Universe is that the shape of $P(k)$ never seems to match observations. This may not be the case with other cosmological parameters $[70,71]$. 


\section{Strings and inflation}

One way of improving upon the previous situation is to consider mixed scenarios: strings and inflation. Recent developments in inflation model building, based on supersymmetry, have produced compelling models in which strings are produced at the end of inflation. In such models the cosmological perturbations are seeded both by the defects and by the quantum fluctuations.

A major drawback of inflationary theories is that they are far-removed from particle physics models. Attempts to improve on this state of affairs have been made recently, resorting to supersymmetry $[72,73,74,75,76$, $77,78]$. In these models one identifies flat directions in the potentials, which are enforced by a (super)symmetry. Such flat directions produce "slow-roll inflation". In order to stop inflation one must tilt the potential, allowing for the fields to roll down. In so-called D-term supersymmetric inflationary scenarios, inflation stops with a symmetry-breaking phase transition, at which a U(1) symmetry is spontaneously broken, leading to the formation of cosmic strings. This is only the most natural of a whole class of models of so-called hybrid inflation. Hence a network of cosmic strings is formed at the end of inflation.

In Figs. (23) and (24) we present power spectra in CMB and CDM produced by a sCDM scenario, by cosmic strings, and by strings plus inflation. We have assumed the traditional choice of parameters, setting the Hubble parameter $H_{0}=50 \mathrm{~km} \mathrm{sec}^{-1} \mathrm{Mpc}^{-1}$, the baryon fraction to $\Omega_{b}=0.05$, and assumed a flat geometry, no cosmological constant, 3 massless neutrinos, standard recombination, and cold dark matter. The inflationary perturbations have a Harrison-Zel'dovich or scale invariant spectrum, and the amount of gravitational radiation (tensor modes) produced during inflation is assumed to be negligible.

We now summarise the results.

- The CMB power spectrum shape in these models is highly exotic. The inflationary contribution is close to being Harrison-Zel'dovich. Hence it produces a flat small $\ell$ CMB spectrum. The admixture of strings, however, imparts a tilt. Depending on $R_{\mathrm{SI}}$ (the ratio of large angle anisotropy due to strings and inflation) one may tune the CMB plateau tilt between 1 and about 1.4, without invoking primordial tilt and inflation produced gravity waves.

- The proverbial inflationary Doppler peaks are transfigured in these scenarios into a thick Doppler bump, covering the region $\ell=200-600$. The height of the peak is similar for sCDM and strings, with standard cosmological parameters. The Doppler bump is modulated by small

undulations, which cannot truly be called secondary peaks. By tuning 
$R_{\mathrm{SI}}$ one may achieve any degree of secondary oscillation softening. This provides a major loophole in the argument linking inflation with secondary oscillations in the CMB power spectrum $[79,56]$. If these oscillations were not observed, inflation could still survive, in the form of the models discussed above.

- In these scenarios the LSS of the Universe is almost all produced by inflationary fluctuations. However COBE scale CMB anisotropies are due to both strings and inflation. Therefore COBE normalized CDM fluctuations are reduced by a factor $\left(1+R_{\mathrm{SI}}\right)$ in strings plus inflation scenarios. This is equivalent to multiplying the sCDM bias by $\sqrt{1+R_{\mathrm{SI}}}$ on all scales, except the smallest, where the string contribution may be non negligible. Given that sCDM scenarios produce too much structure on small scales (too many clusters) this is a desirable feature.

Overall "Strings plus inflation" are interesting first of all as an inflationary model. Its "flat potential" is not the result of a finely tuned coupling constant, but the result of a symmetry. Hence in some sense these models achieve inflation without fine tuning. The only free parameters are the number of inflationary e-foldings, and the scale of symmetry breaking. These parameters also fix the absolute (and therefore relative) normalizations of string and inflationary fluctuations.

The combination of these two scenarios smoothes the hard edges of either separate component, leaving a much better fit to LSS and CMB power spectra. We illustrated this point in this review, but left out a couple of issues currently under investigation which we now summarise.

The CDM power spectrum in these scenarios has a break at very small scales, when string produced CDM fluctuations become dominant over inflationary ones. This aspect was particularly emphasized in [82], and there is some observational evidence in favour of such a break. An immediate implication of this result is that it is easier to form structure at high redshifts [80, 81]. In [83] it is shown that even with Hot Dark Matter, these scenarios produce enough damped Lyman- $\alpha$ systems, to account for the recent high-redshift observations.

Another issue currently under investigation is the timing of structure formation [80]. Active models drive fluctuations at all times, and therefore produce a time-dependence in $P(k)$ different from passive models. The effect is subtle, but works so as to slow down structure formation. Hence for the same normalization nowadays there is more structure at high redshifts in string scenarios $[43,46,47]$. 


\section{Defects and Baryogenesis}

Baryogenesis forms another overlap area between defects in particle physics and cosmology. The goal is to explain the observed asymmetry between matter and antimatter in the Universe. In particular, the objective is to be able to deduce the observed value of the net baryon to entropy ratio at the present time

$$
\frac{\Delta n_{B}}{s}\left(t_{0}\right) \sim 10^{-10}
$$

starting from initial conditions in the very early Universe when this ratio vanishes. Here, $\Delta n_{B}$ is the net baryon number density and $s$ the entropy density.

As pointed out by Sakharov [84], three basic criteria must be satisfied in order to have a chance at explaining the data:

1. The theory describing the microphysics must contain baryon number violating processes.

2. These processes must be $\mathrm{C}$ and $\mathrm{CP}$ violating.

3. The baryon number violating processes must occur out of thermal equilibrium.

As was discovered in the 1970's [85], all three criteria can be satisfied in GUT theories. In these models, baryon number violating processes are mediated by superheavy Higgs and gauge particles. The baryon number violation is visible in the Lagrangian, and occurs in perturbation theory (and is therefore in principle easy to calculate). In addition to standard model $\mathrm{CP}$ violation, there are typically many new sources of $\mathrm{CP}$ violation in the GUT sector. The third Sakharov condition can also be realized: After the GUT symmetry-breaking phase transition, the superheavy particles may fall out of thermal equilibrium. The out-of-equilibrium decay of these particles can thus generate a nonvanishing baryon to entropy ratio.

The magnitude of the predicted $n_{B} / s$ depends on the asymmetry $\varepsilon$ per decay, on the coupling constant $\lambda$ of the $n_{B}$ violating processes, and on the ratio $n_{X} / s$ of the number density $n_{X}$ of superheavy Higgs and gauge particles to the number density of photons, evaluated at the time $t_{d}$ when the baryon number violating processes fall out of thermal equilibrium, and assuming that this time occurs after the phase transition. The quantity $\varepsilon$ is proportional to the $\mathrm{CP}$-violation parameter in the model. In a GUT theory, this $\mathrm{CP}$ violation parameter can be large (order 1), whereas in the standard electroweak theory it is given by the $\mathrm{CP}$ violating phases in the CKM mass matrix and is very small. As shown in [85] it is easily possible to construct models which give the right $n_{B} / s$ ratio after the GUT phase transition (for recent reviews of baryogenesis see [86] and [87]). 
The ratio $n_{B} / s$, however, does not only depend on $\varepsilon$, but also on $n_{X} / s\left(t_{d}\right)$. If the temperature $T_{d}$ at the time $t_{d}$ is greater than the mass $m_{X}$ of the superheavy particles, then it follows from the thermal history in standard cosmology that $n_{X} \sim s$. However, if $T_{d}<m_{X}$, then the number density of $X$ particles is diluted exponentially in the time interval between when $T=m_{X}$ and when $T=T_{d}$. Thus, the predicted baryon to entropy ratio is also exponentially suppressed:

$$
\frac{n_{B}}{s} \sim \frac{1}{g^{*}} \lambda^{2} \varepsilon e^{-m_{X} / T_{d}},
$$

where $g^{*}$ is the number of spin degrees of freedom in thermal equilibrium at the time of the phase transition. In this case, the standard GUT baryogenesis mechanism is ineffective.

However, topological defects may come to the rescue [6]. As was discussed at the beginning of these lecture notes, topological defects will inevitably be produced in the symmetry breaking GUT transition provided they are topologically allowed in that symmetry breaking scheme. The topological defects provide an alternative mechanism of GUT baryogenesis in the following way: Inside of topological defects, the GUT symmetry is restored. In fact, the defects can be viewed as solitonic configurations of $X$ particles. The continuous decay of defects at times after $t_{d}$ provides an alternative way to generate a nonvanishing baryon to entropy ratio. The defects constitute out of equilibrium configurations, and hence their decay can produce a nonvanishing $n_{B} / s$ in the same way as the decay of free $X$ quanta.

The way to estimate the $n_{B} / s$ ratio is as follows: The defect scaling solution gives the energy density in defects at all times. Taking the time derivative of this density, and taking into account the expansion of the Universe, we obtain the loss of energy attributed to defect decay. By energetics, we can estimate the number of decays of individual quanta which the defect decay corresponds to. We can then use the usual perturbative results to compute the resulting net baryon number.

Provided that $m_{X}<T_{d}$, then at the time when the baryon number violating processes fall out of equilibrium (when we start generating a nonvanishing $n_{B}$ ) the energy density in free $X$ quanta is much larger than the defect density, and hence the defect-driven baryogenesis mechanism is subdominant. However, if $m_{X}>T_{d}$, then as indicated in (84), the energy density in free quanta decays exponentially. In contrast, the density in defects only decreases as a power of time, and hence can soon dominate baryogenesis.

One of the most important ingredients in the calculation is the time dependence of $\xi(t)$, the separation between defects. Immediately after the 
phase transition at time $t_{f}$ (when the defect network is formed), the separation is $\xi\left(t_{f}\right) \sim \lambda^{-1} \eta^{-1}$. In the time period immediately following, the time period of relevance for baryogenesis, $\xi(t)$ approaches the Hubble radius according to the equation [23]

$$
\xi(t) \simeq \xi\left(t_{f}\right)\left(\frac{t}{t_{f}}\right)^{5 / 4} .
$$

Using this result to calculate the defect density, we obtain after some algebra

$$
\left.\left.\frac{n_{B}}{s}\right|_{\text {defect }} \sim \lambda^{2} \frac{T_{d}}{\eta} \frac{n_{B}}{s}\right|_{0},
$$

where $n_{B} /\left.s\right|_{0}$ is the unsuppressed value of $n_{B} / s$ which can be obtained using the standard GUT baryogenesis mechanism. We see from (86) that even for low values of $T_{d}$, the magnitude of $n_{B} / s$ which is obtained via the defect mechanism is only suppressed by a power of $T_{d}$. However, the maximum strength of the defect channel is smaller than the maximum strength of the usual mechanism by a geometrical suppression factor $\lambda^{2}$ which expresses the fact that even at the time of defect formation, the defect network only occupies a small fraction of the volume of space.

It has been known for some time that there are baryon number violating processes even in the standard electroweak theory. These processes are, however, non-perturbative. They are connected with the t'Hooft anomaly [88], which in turn is due to the fact that the gauge theory vacuum is degenerate, and that the different degenerate vacuum states have different quantum numbers (Chern-Simons numbers). In theories with fermions, this implies different baryon number. Configurations such as sphalerons [89] which interpolate between two such vacuum states thus correspond to baryon number violating processes.

As pointed out in [90], the anomalous baryon number violating processes are in thermal equilibrium above the electroweak symmetry breaking scale. Therefore, any net baryon to entropy ratio generated at a higher scale will be erased, unless this ratio is protected by an additional quantum number such as a nonvanishing $B-L$ which is conserved by electroweak processes.

However, as first suggested in [91] and discussed in detail in many recent papers (see [92] for reviews of the literature), it is possible to regenerate a nonvanishing $n_{B} / s$ below the electroweak symmetry breaking scale. Since there are $n_{B}$ violating processes and both $\mathrm{C}$ and $\mathrm{CP}$ violation in the standard model, Sakharov's conditions are satisfied provided that one can realize an out-of-equilibrium state after the phase transition. Standard model $\mathrm{CP}$ violation is extremely weak. Thus, it appears necessary to add some sector with extra $\mathrm{CP}$ violation to the standard model in order to obtain an appreciable $n_{B} / s$ ratio. A simple possibility which has been invoked often 
is to add a second Higgs doublet to the theory, with $\mathrm{CP}$ violating relative phases.

The standard way to obtain out-of-equilibrium baryon number violating processes immediately after the electroweak phase transition is [92] to assume that the transition is strongly first order and proceeds by the nucleation of bubbles (note that these are two assumptions).

Bubbles are out-of-equilibrium configurations. Outside of the bubble (in the false vacuum), the baryon number violating processes are unsuppressed, inside they are exponentially suppressed. In the bubble wall, the Higgs fields have a nontrivial profile, and hence (in models with additional $\mathrm{CP}$ violation in the Higgs sector) there is enhanced $\mathrm{CP}$ violation in the bubble wall. In order to obtain net baryon production, one may either use fermion scattering off bubble walls [93] (because of the $\mathrm{CP}$ violation in the scattering, this generates a lepton asymmetry outside the bubble which converts via sphalerons to a baryon asymmetry) or sphaleron processes in the bubble wall itself $[94,95]$. It has been shown that, using optimistic parameters (in particular a large CP violating phase $\Delta \theta_{C P}$ in the Higgs sector) it is possible to generate the observed $n_{B} / s$ ratio. The resulting baryon to entropy ratio is of the order

$$
\frac{n_{B}}{s} \sim \alpha_{W}^{2}\left(g^{*}\right)^{-1}\left(\frac{m_{t}}{T}\right)^{2} \Delta \theta_{C P}
$$

where $\alpha_{W}$ refers to the electroweak interaction strength, $g^{*}$ is the number of spin degrees of freedom in thermal equilibrium at the time of the phase transition, and $m_{t}$ is the top quark mass. The dependence on the top quark mass enters because net baryogenesis only appears at the one-loop level.

However, analytical and numerical studies show that, for the large Higgs masses which are indicated by the current experimental bounds, the electroweak phase transition will unlikely be sufficiently strongly first order to proceed by bubble nucleation. In addition, there are some concerns as to whether it will proceed by bubble nucleation at all (see e.g. [96]).

Once again, topological defects come to the rescue. In models which admit defects, such defects will inevitably be produced in a phase transition independent of its order. Moving topological defects can play the same role in baryogenesis as nucleating bubbles. In the defect core, the electroweak symmetry is unbroken and hence sphaleron processes are unsuppressed [97], provided that the core is sufficiently thick to contain the sphalerons (in recent work [98] it has been shown that this is a rather severe constraint on workable string-mediated electroweak baryogenesis mechanisms). In the defect walls there is enhanced $\mathrm{CP}$ violation for the same reason as in bubble walls. Hence, at a given point in space, a nonvanishing baryon number will be produced when a topological defect passes by. 
Defect-mediated electroweak baryogenesis has been worked out in detail in [8] (see [7] for previous work) in the case of cosmic strings. The scenario is as follows: at a particular point $x$ in space, antibaryons are produced when the front side of the defect passes by. While $x$ is in the defect core, partial equilibration of $n_{B}$ takes place via sphaleron processes. As the back side of the defect passes by, the same number of baryons are produced as the number of antibaryons when the front side of the defect passes by. Thus, at the end a positive number of baryons are left behind. Obviously, the advantage of the defect-mediated baryongenesis scenario is that it does not depend on the order and on the detailed dynamics of the electroweak phase transition.

As in the case of defect-mediated GUT baryogenesis, the strength of defect-mediated electroweak baryogenesis is suppressed by the ratio SF of the volume which is passed by defects divided by the total volume, i.e.

$$
\left.\frac{n_{B}}{s} \sim \mathrm{SF} \frac{n_{B}}{s}\right|_{0},
$$

where $\left.\left(n_{B} / s\right)\right|_{0}$ is the result of (87) obtained in the bubble nucleation mechanism.

A big caveat for defect-mediated electroweak baryogenesis is that the standard electroweak theory does not admit topological defects. However, in a theory with additional physics just above the electroweak scale it is possible to obtain defects (see e.g. [99] for some specific models). The closer the scale $\eta$ of the new physics is to the electroweak scale $\eta_{E W}$, the larger the volume in defects and the more efficient defect-mediated electroweak baryogenesis (however, as pointed out in [98], this effect is counteracted by the fact that the defect velocity at $T=\eta_{E W}$ decreases as $\eta$ decreases). Using the result of (85) for the separation of defects, we obtain (for nonsuperconducting strings)

$$
\mathrm{SF} \sim \lambda\left(\frac{\eta_{E W}}{\eta}\right)^{3 / 2} v_{D}
$$

where $v_{D}$ is the mean defect velocity. Typically [98], the resulting value of $S F$ is too small for string-mediated electroweak baryogenesis to be efficient.

Defect-mediated baryogenesis is much more efficient if the defects are domain walls (since by purely geometrical arguments the factor $S F$ will be much larger). However, as seen in earlier sections of this review, theories with topologically stable domain walls are ruled out because the wall network would overclose the Universe. What one would like is a theory in which a network of walls forms at some time before the electroweak phase transition, remains present until after the transition and then decays. As has recently been realized [100], this goal may be achieved in theories with embedded defects. 
Embedded defects (see [101] for a recent review) in a theory with vacuum manifold $\mathcal{M}$ are exact solutions of the field equations which correspond to topological defects with respect to a submanifold $\mathcal{M}^{\prime}$ of $\mathcal{M}$ of strictly lower dimension. Embedded defects are unstable in the absence of field fluctuations. However [100] if plasma effects provide effective masses to some of the components of the order parameter of the theory such that the space of ground states in the plasma reduces to $\mathcal{M}^{\prime}$, then the embedded defects can be stabilized in the plasma. Embedded strings which can be stabilized in the electric plasma before recombination exist in the standard electroweak theory. It is interesting to investigate if extension of the minimal electroweak theory admit embedded walls. Such walls would then yield ideal candidates for defect-mediated electroweak baryogenesis.

It is important to keep in mind that defects occurring at any scale may have important consequences for baryogenesis which should be considered when exploring the cosmological implications of the models which admit such defects.

\section{References}

1. T.W.G. Kibble, Phys. Rep. 67, 183 (1980).

2. A. Vilenkin, Phys. Rep. 121, 263 (1985).

3. A. Vilenkin and E.P.S. Shellard, 'Strings and Other Topological Defects' (Cambridge Univ. Press, Cambridge, 1994).

4. M. Hindmarsh and T.W.B. Kibble, Rep. Prog. Phys. 58, 477 (1995).

5. R. Brandenberger, Int. J. Mod. Phys. A9, 2117 (1994).

6. R. Brandenberger, A.-C. Davis and M. Hindmarsh, Phys. Lett. B263, 239 (1991).

7. R. Brandenberger, A.-C. Davis and M. Trodden, Phys. Lett. B332, 305 (1994);

R. Brandenberger and A.-C. Davis, Phys. Lett. B308, 79 (1993).

8. R. Brandenberger, A.-C. Davis and M. Trodden, Phys. Lett. B332, 305 (1994);

R. Brandenberger, A.-C. Davis, T. Prokopec and M. Trodden, Phys. Rev. D53, 4257 (1996).

9. K. Dimopoulos, Phys. Rev. D57, 4629 (1998).

10. R. Brandenberger and X. Zhang, Phys. Rev. D59, 081301 (1999).

11. J. MacGibbon and R. Brandenberger, Nucl. Phys. B331, 153 (1990).

12. P. Bhattacharjee, Phys. Rev. D40, 3968 (1989);

P. Bhattacharjee and N. Rana, Phys. Lett. B246, 365 (1990).

13. G. Sigl, D. Schramm and P. Bhattacharjee, Astropart. Phys. 2, 401 (1994).

14. V. Berezinsky, M. Kachelriess and A. Vilenkin, Phys. Rev. Lett. 79, 4302 (1997).

15. T.W.B. Kibble, J. Phys. A9, 1387 (1976).

16. Ya.B. Zel'dovich, Mon. Not. R. astron. Soc. 192, 663 (1980);

A. Vilenkin, Phys. Rev. Lett. 46, 1169 (1981).

17. R. Davis, Phys. Rev. D35, 3705 (1987).

18. N. Turok, Phys. Rev. Lett. 63, 2625 (1989).

19. H. Nielsen and P. Olesen, Nucl. Phys. B61, 45 (1973).

20. D. Kirzhnits and A. Linde, Pis'ma Zh. Eksp. Teor. Fiz. 15, 745 (1972);

D. Kirzhnits and A. Linde, Zh. Eksp. Teor. Fiz. 67, 1263 (1974).

21. C. Bernard, Phys. Rev. D9, 3313 (1974);

L. Dolan and R. Jackiw, Phys. Rev. D9, 3320 (1974);

S. Weinberg, Phys. Rev. D9, 3357 (1974). 
22. R. Brandenberger, Rev. Mod. Phys. 57, 1 (1985).

23. T.W.B. Kibble, Acta Physica Polonica B13, 723 (1982).

24. W. Zurek, Acta Phys. Pol. B24, 1301 (1993).

25. P. Langacker and S.-Y. Pi, Phys. Rev. Lett. 45, 1 (1980).

26. T.W.B. Kibble and E. Weinberg, Phys. Rev. D43, 3188 (1991).

27. T. Vachaspati and A. Vilenkin, Phys. Rev. D30, 2036 (1984).

28. N. Turok, Phys. Scripta T36, 135 (1991).

29. T. Prokopec, A. Sornborger and R. Brandenberger, Phys. Rev. D45, 1971 (1992);

J. Borrill, E. Copeland and A. Liddle, Phys. Lett. 258B, 310 (1991);

A. Sornborger, Phys. Rev. D48, 3517 (1993).

30. Ya.B. Zel'dovich, I. Kobzarev and L. Okun, Zh. Eksp. Teor. Fiz. 67, 3 (1974).

31. Ya.B. Zel'dovich and M. Khlopov, Phys. Lett. 79B, 239 (1978);

J. Preskill, Phys. Rev. Lett. 43, 1365 (1979).

32. M. Barriola and A. Vilenkin, Phys. Rev. Lett. 63, 341 (1989).

33. S. Rhie and D. Bennett, Phys. Rev. Lett. 65, 1709 (1990).

34. N. Turok, Nucl. Phys. B242, 520 (1984).

35. N. Turok and R. Brandenberger, Phys. Rev. D33, 2175 (1986);

A. Stebbins, Ap. J. (Lett.) 303, L21 (1986);

H. Sato, Prog. Theor. Phys. 75, 1342 (1986).

36. J. Silk and V. Vilenkin, Phys. Rev. Lett. 53, 1700 (1984).

37. A. Vilenkin, Phys. Rev. D23, 852 (1981);

J. Gott, Ap. J. 288, 422 (1985);

W. Hiscock, Phys. Rev. D31, 3288 (1985);

B. Linet, Gen. Rel. Grav. 17, 1109 (1985);

D. Garfinkle, Phys. Rev. D32, 1323 (1985);

R. Gregory, Phys. Rev. Lett. 59, 740 (1987).

38. T. Vachaspati, Phys. Rev. Lett. 57, 1655 (1986).

39. A. Stebbins, S. Veeraraghavan, R. Brandenberger, J. Silk and N. Turok, Ap. J. 322, 1 (1987).

40. R. Brandenberger, L. Perivolaropoulos and A. Stebbins, Int. J. of Mod. Phys. A5, 1633 (1990);

L. Perivolaropoulos, R. Brandenberger and A. Stebbins, Phys. Rev. D41, 1764 (1990);

R. Brandenberger, Phys. Scripta T36, 114 (1991).

41. B. Carter, Phys. Rev. D41, 3869 (1990).

42. D. Vollick, Phys. Rev. D45, 1884 (1992);

T. Vachaspati and A. Vilenkin, Phys. Rev. Lett. 67, 1057 (1991).

43. A. Aguirre and R. Brandenberger, Int. J. Mod. Phys. D4, 711 (1995).

44. R. Brandenberger, N. Kaiser, D. Schramm and N. Turok, Phys. Rev. Lett. 59, 2371 (1987).

45. R. Brandenberger, N. Kaiser and N. Turok, Phys. Rev. D36, 2242 (1987).

46. R. Moessner and R. Brandenberger, Mon. Not. R. astron. Soc. 277, 927 (1995).

47. V. Zanchin, J.A.S. Lima and R. Brandenberger, Phys. Rev. D54, 7129 (1996).

48. W. Hu and N. Sugiyama, Ap. J. 444, 480 (1995);

U. Seljak, Ap. J. (Lett.) 435, 87 (1994).

49. W. Hu and M. White, Phys. Rev. D56, 596 (1997).

50. U-L. Pen, U. Seljak and N. Turok, Phys. Rev. Lett. 79, 1611 (1997).

51. C. Contaldi, M. Hindmarsh and J. Magueijo, Phys. Rev. Lett. 82, 679 (1999).

52. C. Contaldi, M. Hindmarsh and J. Magueijo, Phys. Rev. Lett. 82, 2034 (1999).

53. B. Allen et al, Phys. Rev. Lett. 79, 2624 (1997).

54. A. Albrecht, R. Battye and J. Robinson, Phys. Rev. Lett. 79, 4736-4739 (1997).

55. G. Vincent, N. Antunes and M. Hindmarsh, Phys. Rev. Lett. 80, 2277 (1998).

56. A. Albrecht et al., Phys. Rev. Lett. 76, 1413 (1996);

J. Magueijo et al, Phys. Rev. Lett. 76, 2617 (1996).

57. J. Traschen, Phys. Rev. D31, 283 (1985); 
J. Traschen, Phys. Rev. D29, 1563 (1984).

58. J. Robinson and B. Wandelt, Phys. Rev. D53, 618 (1996).

59. L. Abbott and J. Traschen, Ap. J. 302, 39 (1986).

60. H. Kodama and M. Sasaki, Prog. Theor. Phys. Suppl. 78, 1 (1984).

61. N. Turok, U-L. Pen and U. Seljak, Phys. Rev. D58, (1998).

62. J-P. Uzan, N. Deruelle and A. Riazuelo, astro-ph/9810313.

63. A. Smith and A. Vilenkin, Phys. Rev. D36, 990 (1987).

64. M. Sakellariadou and A. Vilenkin, Phys. Rev. D42, 349 (1990).

65. U. Seljak and M. Zaldarriaga, Ap. J. 469, 437 (1997).

66. G. Vincent, M. Hindmarsh, and M. Sakellariadou, Phys.Rev. D55 573 (1997).

67. G. Vincent, M. Hindmarsh, and M. Sakellariadou, Phys.Rev. D56, 637 (1997).

68. L. Perivolaropoulos, Ap. J. 451, 429 (1995).

69. J. Peacock and S. Dodds, Mon. Not. R. astron. Soc. 267, 1020 (1994).

70. A. Albrecht, R. Battye and J. Robinson, Phys. Rev. Lett. 80, 4847 (1998).

71. P. Avelino, E.P.S. Shellard, J.H.P. Wu and B. Allen, Phys. Rev. Lett. 81, 2008 (1998).

72. J. Casas, J. Moreno, C. Muñoz, and M. Quirós, Nucl. Phys. B328, 272 (1989).

73. E. Halyo, Phys. Lett. B387, 43 (1996);

P. Binétruy and G. Dvali, Phys. Lett. B388, 241 (1996).

74. R. Jeannerot, Phys. Rev. D56, 6205 (1997).

75. D. Lyth and A. Riotto, hep-ph/9807278.

76. E. Copeland et al., Phys. Rev. D49, 6410 (1994).

77. I. Tkachev et al., hep-ph/9805209.

78. T. Kanazawa et al., hep-ph/9803293.

79. J. Barrow and A. Liddle, Gen. Rel. Grav. 29, 1503 (1997).

80. C. Steidel et al., astro-ph/9708125.

81. L. Storrie-Lombardi, R. McMahon, and M. Irwin, Mon. Not. R. astron. Soc. 283, L79 (1996).

82. R. Battye and J. Weller, astro-ph/9810203.

83. R. Battye, J. Magueijo and J. Weller, astro-ph/9906093.

84. A. Sakharov, Pisma Zh. Eksp. Teor. Fiz. 5, 32 (1967).

85. M. Yoshimura, Phys. Rev. Lett. 41, 281 (1978);

A. Ignatiev, N. Krasnikov, V. Kuzmin and A. Tavkhelidze, Phys. Lett. 76B, 436 (1978);

S. Dimopoulos and L. Susskind, Phys. Rev. D18, 4500 (1978);

S. Weinberg, Phys. Rev. Lett. 42, 850 (1979);

D. Toussaint, S. Trieman, F. Wilczek and A. Zee, Phys. Rev. D19, 1036 (1979).

86. A. Dolgov, Phys. Rep. 222, 309 (1992).

87. V. Rubakov and M. Shaposhnikov, Usp. Fiz. Nauk 166, 493 (19960, hep$\mathrm{ph} / 9603208$.

88. G. t'Hooft, Phys. Rev. Lett. 37, 8 (1976).

89. N. Manton, Phys. Rev. D28, 2019 (1983);

F. Klinkhamer and N. Manton, Phys. Rev. D30, 2212 (1984).

90. V. Kuzmin, V. Rubakov and M. Shaposhnikov, Phys. Lett. B155, 36 (1985);

P. Arnold and L. McLerran, Phys. Rev. D36, 581 (1987).

91. M. Shaposhnikov, JETP Lett. 44, 465 (1986);

M. Shaposhnikov, Nucl. Phys. B287, 757 (1987);

L. McLerran, Phys. Rev. Lett. 62, 1075 (1989).

92. N. Turok, in 'Perspectives on Higgs Physics', ed. G. Kane (World Scientific, Singapore, 1992);

A. Cohen, D. Kaplan and A. Nelson, Ann. Rev. Nucl. Part. Sci. 43, 27 (1993);

M. Trodden, Rev. Mod. Phys. 71, 1463 (1999);

A. Riotto and M. Trodden, hep-ph/9901362.

93. A. Cohen, D. Kaplan and A. Nelson, Phys. Lett. B245, 561 (1990);

A. Cohen, D. Kaplan and A. Nelson, Nucl. Phys. B349, 727 (1991); 
A. Nelson, D. Kaplan and A. Cohen, Nucl. Phys. B373, 453 (1992);

M. Joyce, T. Prokopec and N. Turok, Phys. Lett. B338, 269 (1994);

M. Joyce, T. Prokopec and N. Turok, Phys. Rev. Lett. 75, 1695 (1995).

94. $\quad$ N. Turok and T. Zadrozny, Phys. Rev. Lett. 65, 2331 (1990);

N. Turok and J. Zadrozny, Nucl. Phys. B358, 471 (1991);

L. McLerran, M. Shaposhnikov, N. Turok and M. Voloshin, Phys. Lett. B256, 451 (1991);

M. Dine, P. Huet, R. Singleton and L. Susskind, Phys. Lett. B257, 351 (1991).

95. A. Cohen, D. Kaplan and A. Nelson, Phys. Lett. B263, 86 (1991);

M. Joyce, T. Prokopec and N. Turok, Phys. Rev. D53, 2958 (1996).

96. M. Gleiser, Phys. Rev. Lett. 73, 3495 (1994);

J. Borrill and M. Gleiser, Phys. Rev. D51, 4111 (1995).

97. W. Perkins, Nucl. Phys. B449, 265 (1995).

98. J. Cline, J. Espinoza, G. Moore and A. Riotto, Phys. Rev. D59, 065014 (1999).

99. M. Trodden, A.-C. Davis and R. Brandenberger, Phys. Lett. B349, 131 (1995).

100. M. Nagasawa and R. Brandenberger, Phys. Lett. B467, 205 (1999).

101. A. Achucarro and T. Vachaspati, hep-ph/9904229. 


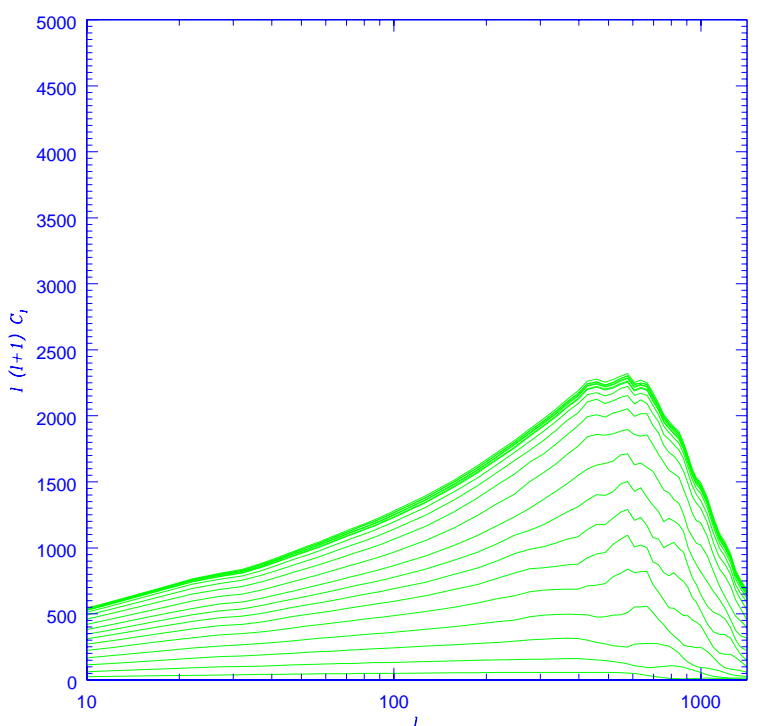

Figure 20. The $C_{\ell}$ spectrum and inferred from 20 eigenmodes. 


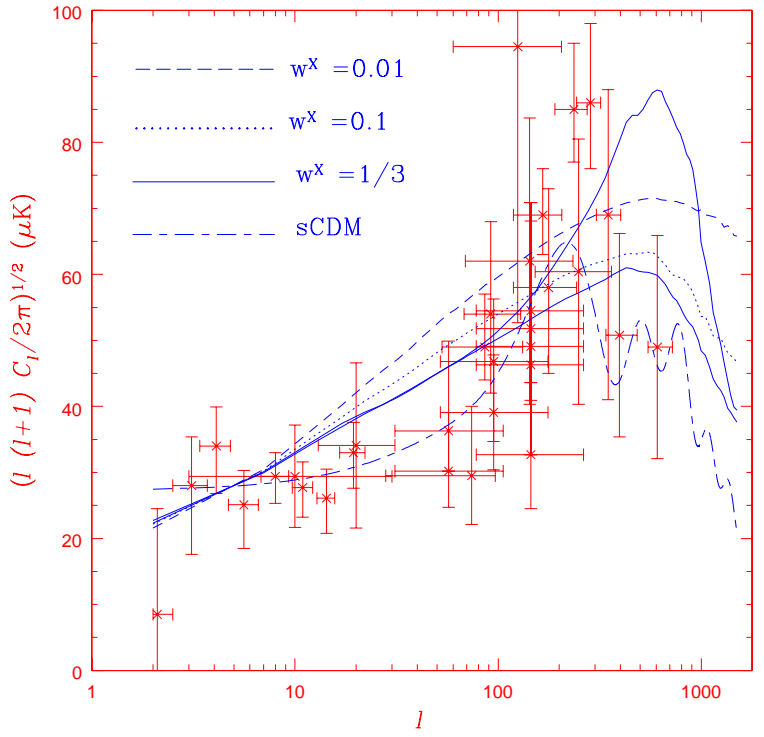

Figure 21. The CMB power spectra predicted by cosmic strings decaying into loop and radiation fluids with $w^{X}=1 / 3,0.1,0.01,0$. We have plotted $\left(\ell(\ell+1) C_{\ell} / 2 \pi\right)^{1 / 2}$ in $\mu K$, and superposed several experimental points. The higher curve corresponding to $w^{X}=1 / 3$ shows what happens if $5 \%$ of the energy goes into the radiation fluid. 


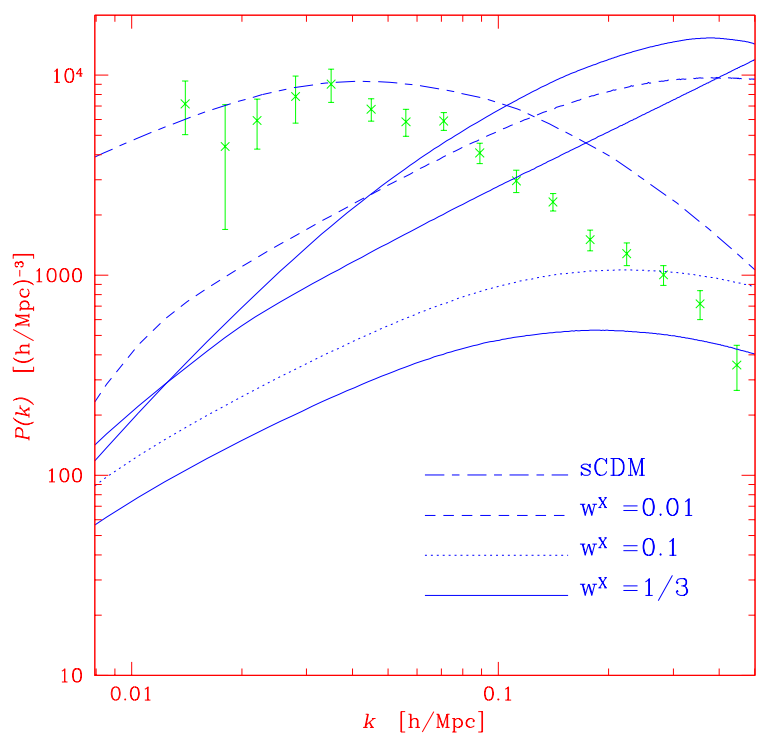

Figure 22. The power spectrum in CDM fluctuations for cosmic strings, with $w^{X}=0.01,0.1,1 / 3$. We plotted also the standard CDM scenario prediction and points inferred by Peacock and Dodds from galaxy surveys. The top $2 w^{X}=1 / 3$ curves correspond to a $5 \%$ transfer into CDM, and a $20 \%$ transfer into baryons (top). 


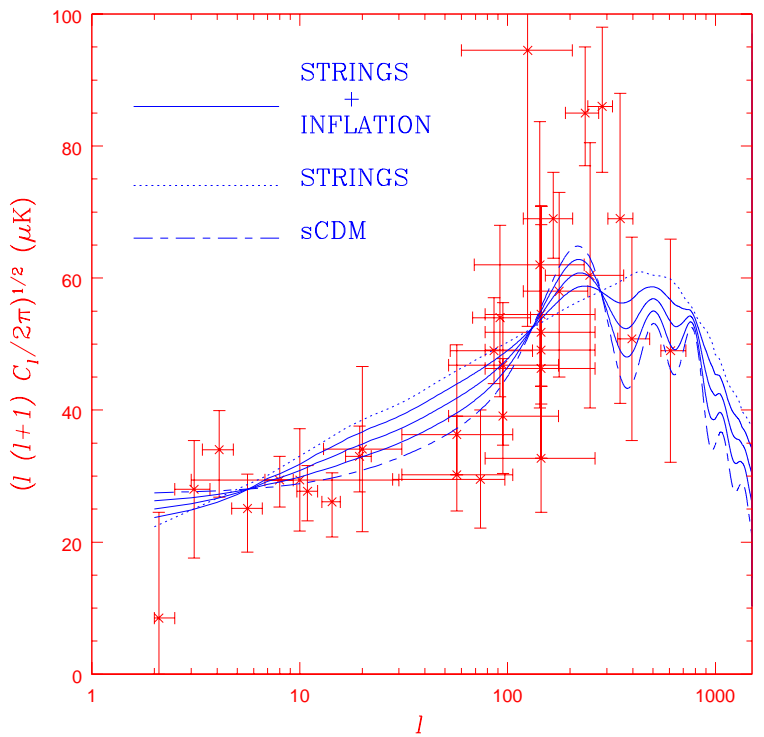

Figure 23. The CMB power spectra predicted by cosmic strings, sCDM, and by inflation and strings with $R_{\mathrm{SI}}=0.25,0.5,0.75$. The large angle spectrum is always slightly tilted. The Doppler peak becomes a thick Doppler bump at $\ell=200-600$, modulated by mild undulations. 


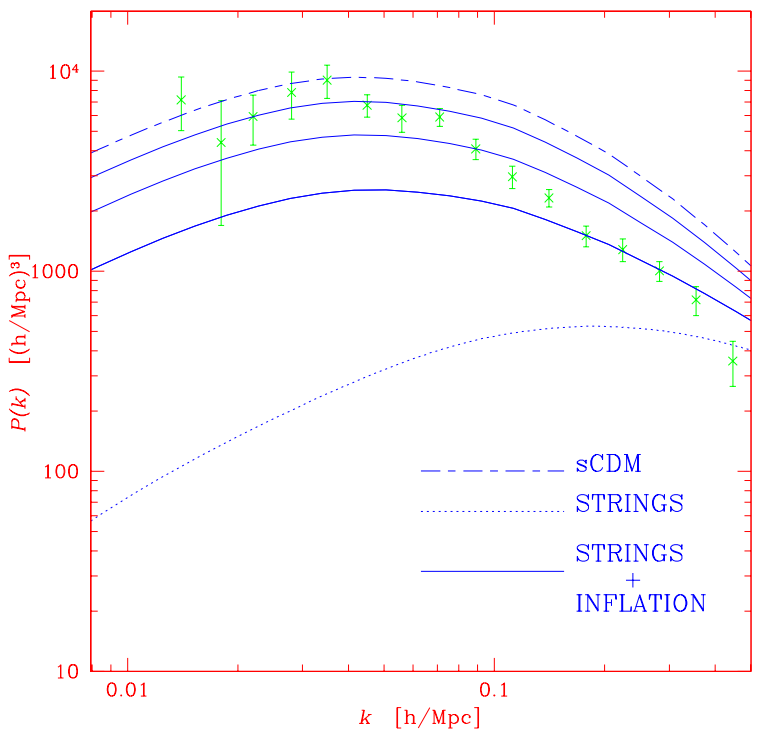

Figure 24. The power spectra in CDM fluctuations predicted by cosmic strings, sCDM, and by inflation and strings with $R_{\mathrm{SI}}=0.25,0.5,0.75$. We have also superposed the power spectrum as inferred from surveys by Peacock and Dodds. 